ESAIM: M2AN 48 (2014) 1725-1755

DOI: $10.1051 / \mathrm{m} 2 \mathrm{an} / 2014017$
ESAIM: Mathematical Modelling and Numerical Analysis

www.esaim-m2an.org

\title{
MONOTONE $(A, B)$ ENTROPY STABLE NUMERICAL SCHEME FOR SCALAR CONSERVATION LAWS WITH DISCONTINUOUS FLUX
}

\author{
Adimurthi ${ }^{1}$, Rajib Dutta ${ }^{1}$, G.D. Veerappa Gowda ${ }^{1}$ and Jérôme Jaffré ${ }^{2}$
}

\begin{abstract}
For scalar conservation laws in one space dimension with a flux function discontinuous in space, there exist infinitely many classes of solutions which are $L^{1}$ contractive. Each class is characterized by a connection $(A, B)$ which determines the interface entropy. For solutions corresponding to a connection $(A, B)$, there exists convergent numerical schemes based on Godunov or Engquist-Osher schemes. The natural question is how to obtain schemes, corresponding to computationally less expensive monotone schemes like Lax-Friedrichs etc., used widely in applications. In this paper we completely answer this question for more general $(A, B)$ stable monotone schemes using a novel construction of interface flux function. Then from the singular mapping technique of Temple and chain estimate of Adimurthi and Gowda, we prove the convergence of the schemes.
\end{abstract}

Mathematics Subject Classification. 35L45, 35L60, 35L65, 35L67.

Received February 8, 2013. Revised September 2, 2013.

Published online September 26, 2014.

\section{INTRODUCTION}

Let $I=[s, S] \subset \mathbb{R}$ be an interval and $f, g$ are continuous functions on $I$. Define the discontinuous flux function

$$
\mathcal{F}(x, u)=H(x) f(u)+(1-H(x)) g(u)
$$

where $H(x)$ is the Heaviside function. Let $u_{0} \in L^{\infty}(\mathbb{R})$ taking values in $I$. Consider the following scalar conservation law:

$$
\begin{array}{lc}
u_{t}+\mathcal{F}(x, u)_{x}=0 & x \in \mathbb{R}, t>0, \\
u(x, 0)=u_{0}(x) & x \in \mathbb{R} .
\end{array}
$$

A function $u \in L_{l o c}^{\infty}\left(\mathbb{R} \times \mathbb{R}^{+}\right)$is called a solution to (1.2) if $u$ satisfies

(i) $u$ is a weak solution, i.e. for all $\phi \in C_{c}^{1}\left(\overline{\mathbb{R} \times \mathbb{R}^{+}}\right), u$ satisfies

$$
\int_{0}^{\infty} \int_{\mathbb{R}}\left[u \phi_{t}+\mathcal{F}(x, u) \phi_{x}\right] \mathrm{d} x \mathrm{~d} t+\int_{\mathbb{R}} u_{0}(x) \phi(x, 0) \mathrm{d} x=0 .
$$

\footnotetext{
Keywords and phrases. Conservation laws, discontinuous flux, Lax-Friedrichs scheme, singular mapping, interface entropy condition, $(A, B)$ connection.

1 TIFR-CAM, PB 6503, Sharadanagar, 560065 Bangalore, India. aditi@math.tifrbng.res.in; rajib@math.tifrbng.res.in; gowda@math.tifrbng.res.in

2 INRIA, BP 105, 78153 Le Chesnay Cedex, France. jerome.jaffre@inria.fr
} 
(ii) For $x \neq 0, u$ satisfies the Kruzkov entropy condition, i.e., $u \in L^{\infty}\left(\mathbb{R} \times \mathbb{R}_{+}\right)$and satisfies for all $l \in \mathbb{R}$ and all non-negative test functions $\phi$ supported away from the interface $\{x=0\}$

$$
\begin{aligned}
& \int_{0}^{\infty} \int_{0}^{\infty}\left[|u-l| \phi_{t}+\operatorname{sgn}(u-l)(f(u)-f(l)) \phi_{x}\right] \mathrm{d} x \mathrm{~d} t \geq 0, \\
& \int_{0}^{\infty} \int_{-\infty}^{0}\left[|u-l| \phi_{t}+\operatorname{sgn}(u-l)(g(u)-g(l)) \phi_{x}\right] \mathrm{d} x \mathrm{~d} t \geq 0 .
\end{aligned}
$$

Basic questions related to this problem is, existence and uniqueness of the solutions and developing a proper numerical scheme to capture them. It is well known that, in general (1.2) may not admit solutions, even if $u_{0}$ is sufficiently smooth. Even if solutions exists, it may not be unique. The development of well-posed theory for the problem (1.2) has been studied extensively in the recent past, see $[1,3,5-9,11,13,15,17,22,24,26]$.

This type of problem appears in numerous models in physics and engineering, for example in modeling of two phase flow in a porous media [13], in sedimentation problem [11,12] and in traffic flow [20].

Under suitable assumptions on $f$ and $g$, this problem admits a solution. In general several solutions may exist. To find a physically relevant unique solution, one has to impose an extra condition called an interface entropy condition which was first introduced in [1] at the interface $\{x=0\}$. Later, a family of interface entropy conditions (called $(A, B)$ entropy) were introduced in [5] and showed that in each class there exists a unique solution.

Assume that the fluxes $f$ and $g$ are either bell-shaped or inverted bell-shaped functions having common end points. Under this assumption, basically there are three known methods to study equation (1.2) with $(A, B)$ interface entropy condition.

(a) The Hamilton-Jacobi method: Hamilton-Jacobi method was used in $[1,4]$ for strictly convex fluxes $f$ and $g$, where an explicit formulae for the solutions were obtained. Moreover, $L^{1}$-contractivity of the solution was shown.

(b) the vanishing Viscosity method: in [6], by using vanishing viscosity method, existence and uniqueness of $(A, B)$ interface entropy condition was studied.

(c) Numerical schemes: in [5,9] the Godunov and Engquist-Osher type schemes were constructed for each $(A, B)$-interface entropy. Convergence of the scheme and uniqueness of the solution satisfying $(A, B)$ interface entropy condition were shown.

In general, a constant data result in a non-constant solution and hence one can not expect a total variation diminishing scheme. Moreover it was an open problem whether it is possible to get a total variation bound. Recently a counter example was constructed in [2] to show that in any neighbourhood of the interface $\{x=0\}$, the total variation becomes infinity. Hence the convergence analysis becomes difficult. This was over come by using the singular mapping technique due to Temple [23] which has been adopted in $[3,5,25]$ for Godunov and Engquist-Osher type schemes.

Goal of this paper is to extend the numerical schemes originating from monotone schemes from a continuous flux case to a discontinuous flux case. This can be done by

(1) Construction of an interface flux function for a given $(A, B)$ connection.

(2) Using (1), construction of convergent monotone $(A, B)$ stable numerical schemes.

Basic idea is to achive (1) as follows. First we consider the undercompressive intersection case, that is, $f$ and $g$ intersect at $c$ with $f^{\prime}(c)>0, g^{\prime}(c)<0$. We construct an interface flux function $l$ by using the decreasing portion of $f$ and the increasing portion of $g$ above the connection (see Fig. 1). The same construction is not possible if it is not undercompressive intersection case because the interface flux function $l$ either is multivalued or not stable with respect to $(A, B)$. To overcome this difficulty we use the translation invariant property of equation (1.2). Next we generate monotone $(A, B)$ stable entropy numerical schemes from this interface flux function $l$. To study the convergence analysis, a singular mapping technique is used, unlike in the case of 
Godunov or Engquist-Osher type schemes,singular mapping techniques become difficult for general schemes. To overcome this difficulty we use the chain estimates of Adimurthi and Gowda ([19], p. 209).

Central schemes like Lax-Friedrichs schemes do not use solution of Riemann problems and its numerical fluxes are calculated by point evaluation. This makes them very simple and easy to implement. Very much useful to problems where the Riemann solution is not known or is too costly to be used. Eventhough Lax-Friedrichs scheme is diffussive compared to other upwind schemes, it is used to construct FORCE scheme and high resolution schemes. In [21], a high resolution schemes which is based on the staggered form of the Lax-Friedrichs scheme is constructed and gives good resolution as of upwind schemes. Lax-Friedrichs scheme is the forerunner for such central schemes and therefore it is important to extend it to discontinuous flux case. Lax-Friedrichs scheme for discontinuous flux, considered in [16] converges to the solution obtained via vanishing viscosity method and captures the particular connection,for example in a case where $f$ and $g$ intersects at $\alpha$ with $f^{\prime}(\alpha)>0$ and $g^{\prime}(\alpha)<0$ (under compressive intersection case). Lax-Friedrichs scheme [16] captures only $A=B=\alpha$ connection. But different $(A, B)$ entropy solution occurs in a natural way in the applications [9]. On the other hand, the Lax-Friedrichs scheme presented here captures all $(A, B)$ interface entropy solution.

This paper is organized as follows: in Section 2, we recall the basic definition of monotone flux, properties of $(A, B)$-interface entropy condition and state the main results. In Section 3 we motivate and construct the interface flux function. Section 4 is devoted to the proof of convergence of the monotone schemes. These schemes are conservative, monotone but not consistent except at the end points. As a result, maximum principle is not satisfied but we have only $\mathrm{L}^{\infty}$ stability as in [3]. Singular mapping technique and chain estimate is used to obtain a total variation bound in the singular variable. Following the idea of Crandall-Majda [10] we show that the numerical scheme satisfy the $(A, B)$-interface entropy condition. Finally in Section 5 , numerical experiments are presented to compare the solution of Godunov scheme and Lax-Friedrichs scheme for the two phase flow in porous media.

\section{Preliminaries}

Let $I=[s, S]$ and $\operatorname{Lip}(I)$ denote the space of all Lipschitz continuous functions on $I$. For $f \in \operatorname{Lip}(I)$, denote $L(f)$ the Lipschitz constant of $f$. Define

$$
\mathbb{F}(I)=\{f \in \operatorname{Lip}(I): f \text { has no local maxima in the interior of } I\} .
$$

Definition 2.1 (monotone Numerical flux). Let $k \in \mathbb{F}(I)$. Then $K \in \operatorname{Lip}(I \times I)$ is said to be a monotone numerical flux corresponding to $k$ if

(i) $K(a, a)=k(a)$.

(ii) $a \mapsto K(a, b)$ is a non-decreasing function and $b \mapsto K(a, b)$ is a non-increasing function.

\section{Important examples.}

(1) Godunov numerical flux $K^{G}$ :

$$
K^{G}(a, b)= \begin{cases}\min _{[a, b]} k(\theta) & \text { if } a \leq b, \\ \max _{[b, a]} k(\theta) & \text { if } b \leq a .\end{cases}
$$

(2) Engquist-Osher numerical flux $K^{E O}$ :

$$
K^{E O}(a, b)=\frac{1}{2}\left(k(a)+k(b)-\int_{a}^{b}\left|k^{\prime}(\theta)\right| \mathrm{d} \theta\right) .
$$


(3) Lax-Friedrichs numerical flux $K^{L F}$ : Let $\lambda\left|k^{\prime}(\theta)\right| \leq 1$ for all $\theta$, then

$$
\begin{aligned}
K^{L F}(a, b) & =\frac{1}{2}\left(k(a)+k(b)-\frac{b-a}{\lambda}\right) \\
& =\frac{1}{2}\left(k(a)+k(b)-\frac{1}{\lambda} \int_{a}^{b}\left|\psi^{\prime}(\theta)\right| \mathrm{d} \theta\right)
\end{aligned}
$$

where $\psi(\theta)=\theta$.

Remark 2.2. Introduction of this $\psi$ will play an important role in the construction of interface numerical flux for Lax-Friedrichs scheme corresponding to the discontinous flux.

Construction of the numerical scheme. Throughout the paper we assume that $f$ and $g$ satisfies the following.

(H) Hypothesis. Let $f, g \in \mathbb{F}(I)$ and $F, G \in \operatorname{Lip}(I \times I)$. Assume that

(i) $f(s)=g(s), f(S)=g(S)$.

(ii) $F$ and $G$ are monotone numerical fluxes of $f$ and $g$ respectively.

Before the construction of numerical schemes, let us recall the definition of $(A, B)$ interface entropy condition.

Interface entropy conditions. Let $f\left(\theta_{f}\right)=\min _{I} f, g\left(\theta_{g}\right)=\min _{I} g$. Then

Definition 2.3. $(A, B) \in(I \times I)$ is called a connection with respect to $f$ and $g$ if

(i) $f(B)=g(A)$,

(ii) $A \leq \theta_{g}, B \geq \theta_{f}$.

Next we define the points $\bar{A}$ and $\bar{B}$ such that $g(\bar{A})=g(A), f(\bar{B})=f(B), \bar{A} \geq \theta_{g}, \bar{B} \leq \theta_{f}$.

Definition 2.4. Let $(A, B)$ be a connection and $u$ be a solution of $(1.2)$ having traces $u^{ \pm}(t)=u(0 \pm, t)$. Then $u$ is said to satisfy the $(A, B)$ interface entropy condition if

$$
\text { meas }\left\{t>0: u^{+}(t) \geq \bar{B}, u^{-}(t) \leq \bar{A},\left(u^{-}(t), u^{+}(t)\right) \neq(A, B),(\bar{A}, B),(A, \bar{B}),(\bar{A}, \bar{B})\right\}=0 .
$$

Numerical schemes. Let $F, G$ and $\bar{F}$ be monotone numerical fluxes. Associated to these,we will define the numerical scheme for (1.2) connecting $F$ and $G$ via $\bar{F}$. This $\bar{F}$ is called as interface numerical flux.

Let $h>0$ and define the space grid points $x_{i}$ as follows:

$$
x_{i+\frac{1}{2}}=i h .
$$

For the time discretization the time step is $\Delta t>0$ and let $t_{n}=n \triangle t$. We also define

$$
\lambda=\frac{\triangle t}{h}, I_{i}=\left[x_{i-\frac{1}{2}}, x_{i+\frac{1}{2}}\right), R_{i, n}=I_{i} \times\left[t_{n}, t_{n+1}\right) .
$$

For an initial data $u_{0} \in L^{\infty}(\mathbb{R}, I)$, define

$$
u_{i}^{0}=\frac{1}{\left|I_{i}\right|} \int_{I_{i}} u_{0}(\theta) \mathrm{d} \theta .
$$

Define the finite volume scheme for $n \geq 0$, as follows:

$$
\begin{array}{lll}
u_{i}^{n+1}=u_{i}^{n}-\lambda\left(F\left(u_{i}^{n}, u_{i+1}^{n}\right)-F\left(u_{i-1}^{n}, u_{i}^{n}\right)\right) & \text { if } & i \geq 2 \\
u_{i}^{n+1}=u_{i}^{n}-\lambda\left(G\left(u_{i}^{n}, u_{i+1}^{n}\right)-G\left(u_{i-1}^{n}, u_{i}^{n}\right)\right) & \text { if } & i \leq-1 \\
u_{1}^{n+1}=u_{1}^{n}-\lambda\left(F\left(u_{1}^{n}, u_{2}^{n}\right)-\bar{F}\left(u_{0}^{n}, u_{1}^{n}\right)\right) & & \\
u_{0}^{n+1}=u_{0}^{n}-\lambda\left(\bar{F}\left(u_{0}^{n}, u_{1}^{n}\right)-G\left(u_{-1}^{n}, u_{0}^{n}\right)\right) . & &
\end{array}
$$


Associated to the discretization, define the singular mapping total variation STV of $u_{0}$ by

$$
\begin{aligned}
\operatorname{STV}\left(u_{0}\right)= & \sup _{h>0}\left\{\sum_{i=-\infty}^{-1}\left|G\left(u_{i}^{0}, u_{i+1}^{0}\right)-G\left(u_{i-1}^{0}, u_{i}^{0}\right)\right|+\sum_{i=2}^{\infty}\left|F\left(u_{i}^{0}, u_{i+1}^{0}\right)-F\left(u_{i-1}^{0}, u_{i}^{0}\right)\right|\right. \\
& \left.+\left|F\left(u_{1}^{0}, u_{2}^{0}\right)-\bar{F}\left(u_{0}^{0}, u_{1}^{0}\right)\right|+\left|\bar{F}\left(u_{0}^{0}, u_{1}^{0}\right)-G\left(u_{-1}^{0}, u_{0}^{0}\right)\right|\right\} .
\end{aligned}
$$

and the piecewise constant function $u_{h}$ by

$$
u_{h}(x, t)=u_{i}^{n}(x, t) \quad \text { if }(x, t) \in R_{i, n} .
$$

It is easy to see that if $u_{0} \in \mathrm{BV}(\mathbb{R})$ then $\operatorname{STV}\left(u_{0}\right) \leq C\left\|u_{0}\right\|_{B V}$, where $C$ is a constant depending only on the Lipschitz constant of $F$ and $G$.

Next we state the main theorem regarding the convergence result.

Theorem 2.5. Let F, G satisfy the hypothesis $(\mathbf{H})$. Assume that $u_{0}$ and $\lambda$ satisfies

(i) CFL conditions: $\lambda L(F) \leq 1, \lambda L(G) \leq 1, \lambda L(\bar{F}) \leq 1$ where $L(k)$ denotes the Lipschitz constant of $k$.

(ii) $\operatorname{STV}\left(u_{0}\right)<\infty$.

Then for fixed $\lambda$, there exist a subsequence $h_{k} \rightarrow 0$ such that $u_{h_{k}}$ converges to $u$ in $L_{l o c}^{1}$ to a solution of (1.2).

Goal. Given $F, G$ and $(A, B)$ entropy condition, the goal of this paper is to construct a monotone numerical flux $\bar{F}$ such that

(i) $\bar{F}$ is an interface numerical flux.

(ii) $\bar{F}=F=G$ if $f=g$ and $A=B=\theta_{f}=\theta_{g}$.

(iii) the associated scheme converges to an $(A, B)$ entropy solution.

In order to achieve this goal we need the following stability condition.

Definition 2.6. $\bar{F}$ is said to satisfy $(A, B)$ stability condition if

$$
\bar{F}(A, B)=g(A)=f(B) .
$$

Then we have the following theorem,

Theorem 2.7. Let $\bar{F}$ satisfies $(A, B)$ stability condition (2.10). Let $u$ be a solution of (1.2) obtained in Theorem 2.1 such that the trace of $u$ exists at the interface. Then u satisfies interface entropy condition (2.5). Furthermore the solution is unique.

In order to prove this theorem, we reformulate the interface entropy condition (2.5) based on $[1,5,9]$ as follows.

Definition 2.8. Let $(A, B)$ be a connection and $u \in \mathrm{L}_{l o c}^{1}\left(\mathbb{R} \times \mathbb{R}^{+}\right)$having traces $u^{ \pm}(t)$ for a.e. $t>0$. Then define

$$
I(u, t)=\left(g\left(u^{-}(t)\right)-g(A)\right) \operatorname{sign}\left(u^{-}(t)-A\right)-\left(f\left(u^{+}(t)\right)-f(B)\right) \operatorname{sign}\left(u^{+}(t)-B\right) .
$$

Then we have the following lemma.

Lemma 2.9. Let $u$ be a solution of (1.2) having traces $u^{ \pm}(t)$ for a.e. $t>0$. Then $u$ satisfies $(A, B)$ interface entropy condition if and only if for a.e. $t>0$,

$$
I(u, t) \geq 0
$$


Proof. We prove the lemma for undercompressive case. The other cases follows similarly. Define $L_{1}=\left\{u^{+}(t) \geq\right.$ $\bar{B}\}, L_{2}=\left\{u^{-}(t) \leq \bar{A}\right\}, L_{3}=\left\{\left(u^{-}(t), u^{+}(t)\right) \neq(A, B),(\bar{A}, B),(A, \bar{B}),(\bar{A}, \bar{B})\right\}$. If $t \notin L_{3}$, then it is easy to see $I(u, t)=0$. Assume that $t \notin L_{1}$, then $u^{+}<\bar{B}$. Since $f\left(u^{+}\right)=g\left(u^{-}\right)$, hence either $u^{-}>\bar{A}$ or $u^{-}<A$. If $u^{-}<A$, then $I(u, t)=-\left(g\left(u^{-}\right)-g(A)\right)+(f(u)-f(B))=0$. If $u^{-}>\bar{A}$, then $I(u, t)=\left(g\left(u^{-}\right)-g(A)\right)+\left(f\left(u^{+}\right)-f(B)\right) \geq 0$. Similarly if $t \notin L_{2}$, then $I(u, t) \geq 0$. Therefore $I(u, t) \geq 0$.

Next we assume that $I(u, t) \geq 0$. We also assume that $u^{-} \leq \bar{A}$ and $u^{+} \geq \bar{B}$. Since $f\left(u^{+}\right)=g\left(u^{-}\right)$, hence either $A \leq u^{-} \leq \bar{A}$ and $\bar{B} \leq u^{+} \leq B$ or $u^{-}<A$ and $u^{+}>B$. If $A \leq u^{-} \leq \bar{A}$ and $\bar{B} \leq u^{+} \leq B$

$$
0 \leq I(u, t)=\left(g\left(u^{-}\right)-g(A)\right)+\left(f\left(u^{+}\right)-f(B)\right)<0
$$

which gives contradiction if $\left(u^{-}(t), u^{+}(t)\right) \neq(A, B),(\bar{A}, B),(A, \bar{B}),(\bar{A}, \bar{B})$. Again if $u^{-}<A$ and $u^{+}>B$, then

$$
0 \leq I(u, t)=-\left(g\left(u^{-}\right)-g(A)\right)-\left(f\left(u^{+}\right)-f(B)\right)<0
$$

which gives a contradiction. This completes the proof of the lemma.

Before going to the proof of the main theorem, first let us describe the construction of interface numerical fluxes $\bar{F}$ satisfying the $(A, B)$ stability condition.

\section{Construction of interface flux satisfying the $(A, B)$ Stablity condition}

Let $(A, B)$ be a connection and $\bar{F}^{G}(a, b)$ denote the Godunov interface flux defined in [3]. The basic question is, how to construct a interface function $l \in \operatorname{Lip}(I)$ such that $\bar{F}^{G}(a, b)=L^{G}(a, b)$ for all $a, b \in I$ where $L^{G}(a, b)$ denote the Godunov numerical flux associated to $l$ ? If the answer to this question is affirmative then using this interface flux function "l", one can generate other types of numerical schemes. In general,this is not true. But with a certain types of translation, one can construct "l" satisfying the above condition. Then the goal of this section is to use this " $l$ " to construct other $(A, B)$ stable numerical fluxes.

In order to understand this, first consider the case of undercompressive intersection. That is there exist $\theta_{f} \leq c \leq \theta_{g}$ such that

$$
f(c)=g(c)
$$

Hence let $\bar{B} \leq \theta_{f}, \bar{A} \geq \theta_{g}$ such that

$$
f(\bar{B})=f(B)=g(A)=g(\bar{A}) .
$$

Now define the interface flux function $l$ by

$$
l(\theta)=\left\{\begin{array}{lll}
f(\theta) & \text { if } & \theta \leq \bar{B} \\
f(B) & \text { if } & \bar{B} \leq \theta \leq \bar{A} \\
g(\theta) & \text { if } & \theta \geq \bar{A} .
\end{array}\right.
$$

It is easy to verify that (proof is given in Lem. 3.1)

$$
\bar{F}^{G}(a, b)=L^{G}(a, b) .
$$

Now the question is, can we use $l$ to generate other types of monotone numerical fluxes like Engquist-Osher and Lax-Friedrichs? Infact it is not, for example if we denote $L^{L F}$ to be the Lax-Friedrichs numerical flux associated to $l$, then it is given by

$$
L^{L F}(a, b)=\frac{1}{2}\left(l(a)+l(b)-\frac{1}{\lambda}(b-a)\right) .
$$

Hence

$$
L^{L F}(A, B)=\frac{1}{2}\left(l(A)+l(B)-\frac{1}{\lambda}(B-A)\right)=f(B)-\frac{1}{2 \lambda}(B-A) .
$$

Hence $L^{L F}(A, B)=f(B)$ if and only if $B=A$. Hence $L^{L F}$ need not be $(A, B)$ stable. 


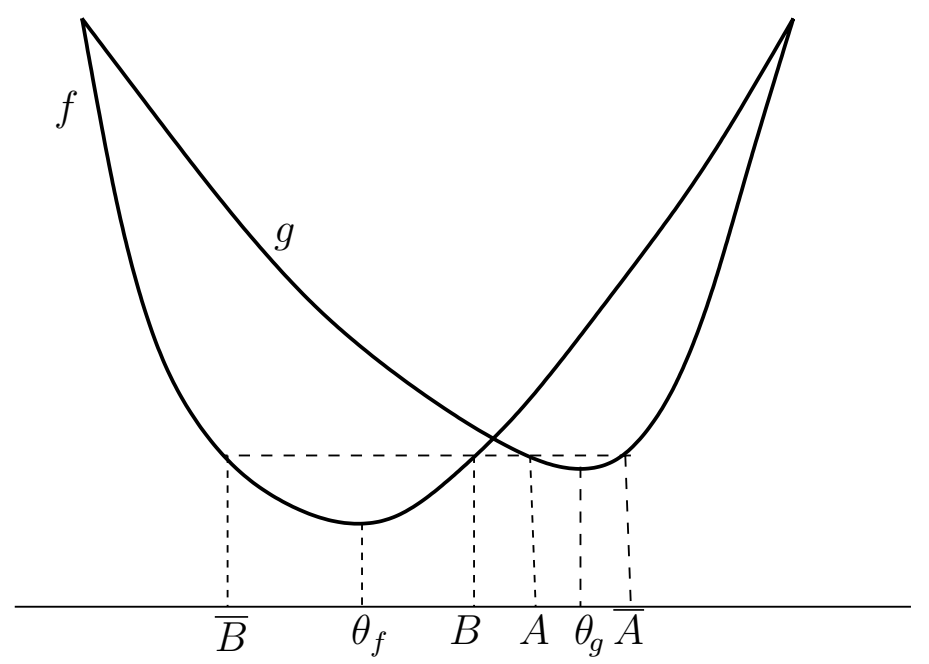

FiguRE 1. Undercompressive intersection case.

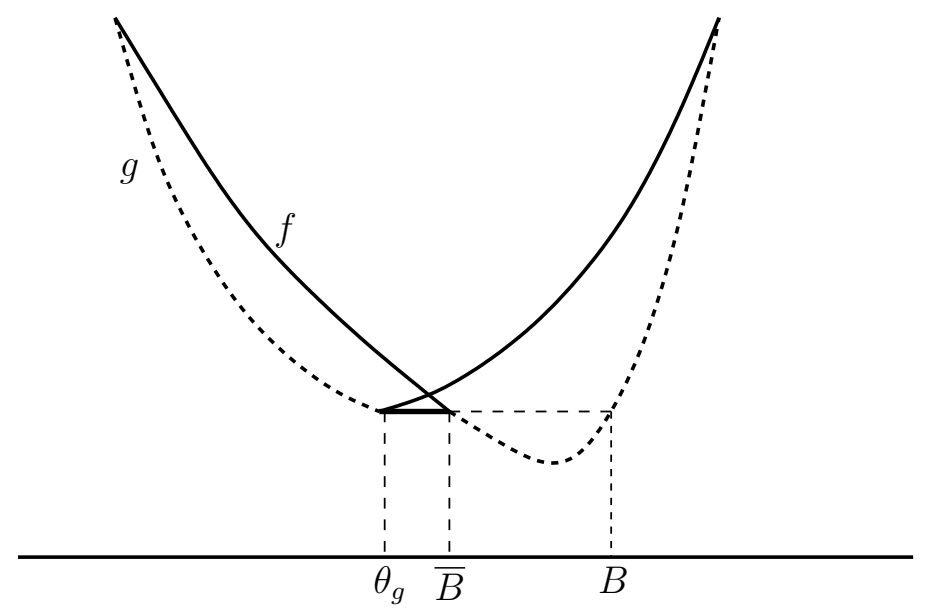

FiguRE 2. Overcompressive intersection case.

If $f$ and $g$ do not have undercompressive intersection then in general, associated interface flux $l$ is not single valued. Therefore we face two difficulties namely $l$ may not be defined. Even if it is defined then the associated numerical flux may not be $(A, B)$ stable.

In the next section we overcome these difficulties and construct a general interface monotone fluxe satisfying $(A, B)$ stability.

Definition 3.1. Let $\bar{B} \leq \theta_{f}, \bar{A} \geq \theta_{g}$ be such that

$$
f(B)=f(\bar{B})=g(A)=g(\bar{A}) .
$$

Then

(i) Undercompressive: $f$ and $g$ said to have undercompressive with respect to $(A, B)$ if

$$
\bar{B} \leq \bar{A}, \quad A, B \in[\bar{B}, \bar{A}] .
$$




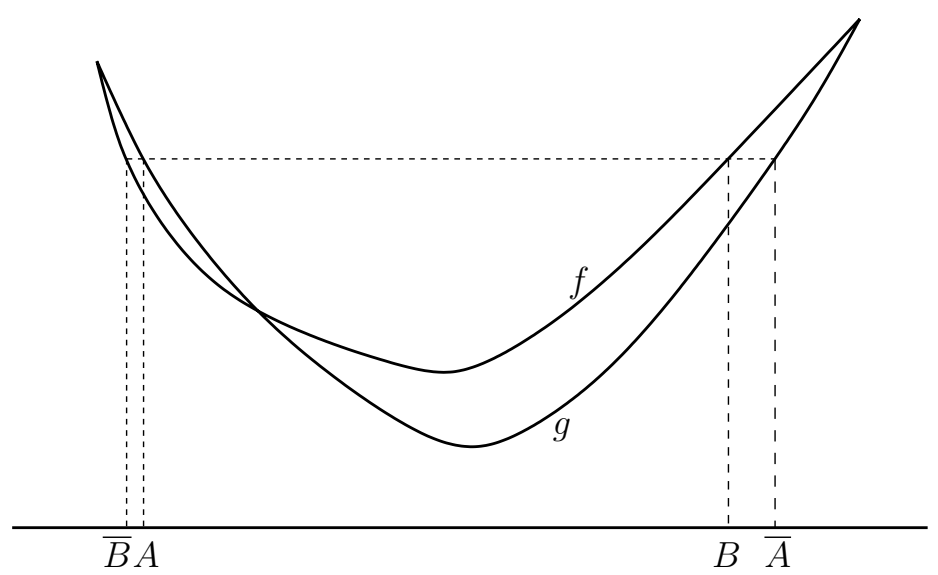

FiguRE 3. Undercompressive intersection case.

(ii) Overcompressive: if $(f, g)$ is not undercompressive with respect to $(A, B)$.

Remark 3.2. The pair $(f, g)$ are undercompressive need not imply that there exists a $c \in\left(\theta_{f}, \theta_{g}\right)$ such that $f(c)=g(c)$, see Figure 3 .

\subsection{Translation invariance}

Let $\delta_{1}, \delta_{2} \in \mathbb{R}$ and define the linear maps $\alpha_{1}$ and $\alpha_{2}$ by $\alpha_{1}(\theta)=\theta+\delta_{1}, \alpha_{2}(\theta)=\theta+\delta_{2}$. Let $I_{j}=\alpha_{j}(I)$ for $j=1,2$ and $\bar{g}(\theta)=g\left(\theta-\delta_{1}\right), \theta \in I_{1}, \bar{f}(\theta)=f\left(\theta-\delta_{2}\right), \theta \in I_{2}$. Let $u$ be a function of $(x, t)$ with values in $I$, then define

$$
\bar{u}(x, t)= \begin{cases}u(x, t)+\delta_{1} & \text { if } \quad x<0 \\ u(x, t)+\delta_{2} & \text { if } \quad x>0\end{cases}
$$

Let

$$
\overline{\mathcal{F}}(x, \bar{u})=H(x) \bar{f}(\bar{u})+(1-H(x)) \bar{g}(\bar{u}),
$$

and

$$
\bar{u}_{0}(x)= \begin{cases}u_{0}(x)+\delta_{1} & \text { if } \quad x<0 \\ u_{0}(x)+\delta_{2} & \text { if } \quad x>0\end{cases}
$$

Then we have the following translation invariant property.

Proposition 3.3. $u$ is a solution of (1.2) if and only if $\bar{u}$ is a solution of

$$
\begin{array}{ll}
\bar{u}_{t}+\overline{\mathcal{F}}(x, \bar{u})_{x}=0 & x \in \mathbb{R}, t>0, \\
\bar{u}(x, 0)=\bar{u}_{0}(x) & x \in \mathbb{R} .
\end{array}
$$

Observe that $\bar{f}$ and $\bar{g}$ are defined on different intervals $I_{2}$ and $I_{1}$ respectively and range of $\overline{u_{0}}$ for $x<0$ and $x>0$ are in the interval $I_{1}$ and $I_{2}$ respectively. Using this invariance property we define the interface flux function $l$ as follows. 


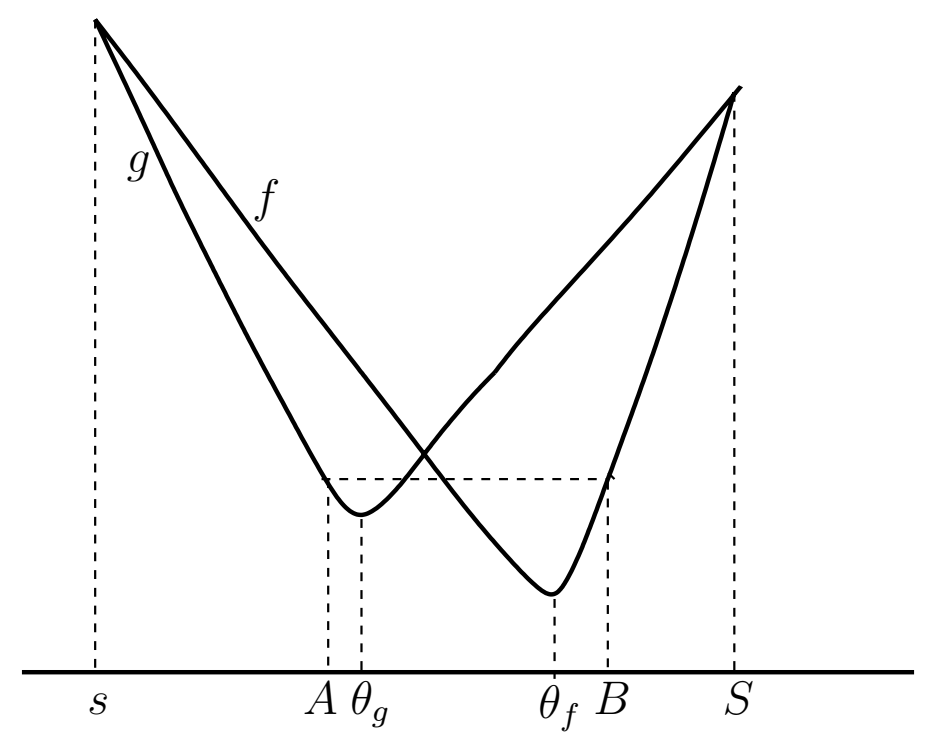

FiguRE 4. Overcompressive intersection case.

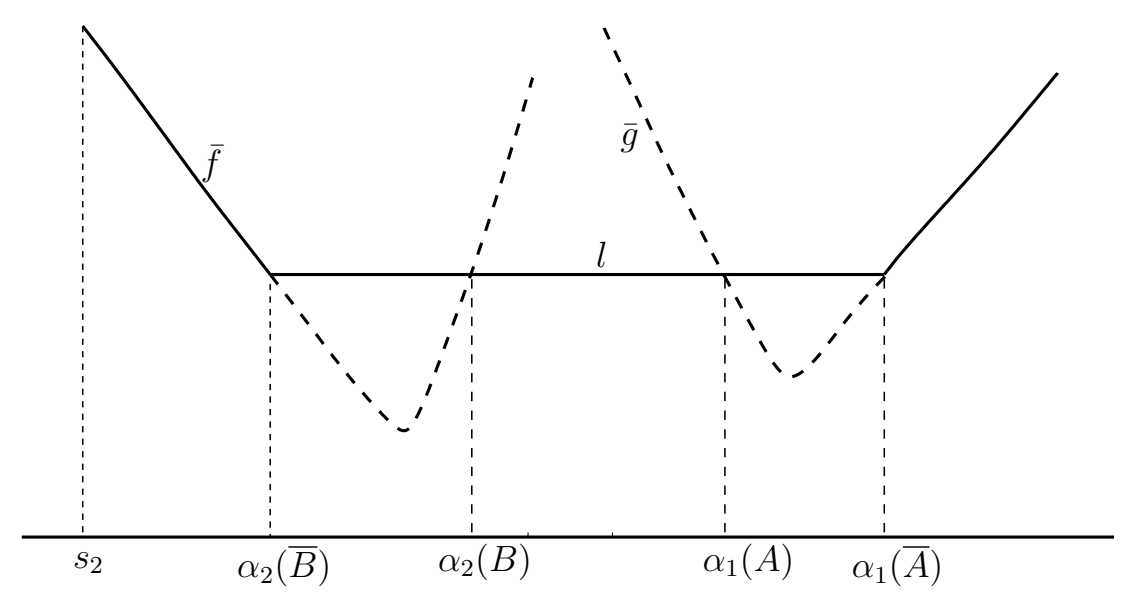

FiguRE 5. Overcompressive intersection case: $l$ function.

\subsection{Interface flux $l$ for $(A, B)$ connection}

We consider the following cases.

Undercompressive case. In this case let $\alpha_{1}(\theta)=\theta$ and $\alpha_{2}(\theta)=\theta$, i.e., $\delta_{1}=0=\delta_{2}$. Let $(A, B)$ be a given connection. Define the function $l:[s, S] \rightarrow \mathbb{R}$ by

$$
l(\theta)=\left\{\begin{array}{lll}
f(\theta) & \text { if } & s \leq \theta \leq \bar{B} \\
f(\bar{B})=g(A) & \text { if } & \theta \in[\bar{B}, \bar{A}] \\
g(\theta) & \text { if } & A \leq \theta \leq S .
\end{array}\right.
$$


With the above construction, we now define interfaces fluxes corresponds to Godunov, Engquist-Osher and Lax-Friedrichs. Let $\psi \in \operatorname{Lip}([s, S])$ such that

$$
\psi=0 \quad \text { on }[\bar{B}, \bar{A}] \text {. }
$$

Let $L^{G}$ be the Godunov numerical flux associated to $l$. For $a, b, \in I=[s, S]$, define

$$
\bar{F}^{G}(a, b)=L^{G}(a, b)
$$

and

$$
\bar{F}^{\psi}(a, b)=\frac{1}{2}\left(l(a)+l(b)-\int_{a}^{b}\left|\psi^{\prime}(\theta)\right| \mathrm{d} \theta\right) .
$$

Overcompressive case. Let $\delta_{1}, \delta_{2} \in \mathbb{R}$ be such that $\delta_{2}-\delta_{1}=B-A$. Then $s-\delta_{2} \leq \bar{B}-\delta_{2} \leq B-\delta_{2}=A-\delta_{1} \leq$ $\bar{A}-\delta_{1} \leq S-\delta_{1}$. Let $\alpha_{1}(\theta)=\theta+\delta_{1}, \alpha_{2}(\theta)=\theta+\delta_{2}$. Then define the interface flux $l$ on $I_{0}=\left[s-\delta_{2}, S-\delta_{1}\right]$ by

$$
l(\theta)= \begin{cases}\bar{f}(\theta)=f\left(\alpha_{2}(\theta)\right) & \text { if } \quad \theta \leq \bar{B}-\delta_{2} \\ f(B)=g(A) & \text { if } \quad \bar{B}-\delta_{2} \leq \theta \leq \bar{A}-\delta_{1} \\ \bar{g}(\theta)=g\left(\alpha_{1}(\theta)\right) & \text { if } \quad \bar{A}-\delta_{1} \leq \theta \leq S-\delta_{1} .\end{cases}
$$

Now $l\left(\bar{B}-\delta_{2}\right)=f(\bar{B})=g(\bar{A})=l\left(\bar{A}-\delta_{1}\right)$, hence $l \in \operatorname{Lip}\left(I_{0}\right)$.

Now define the numerical fluxes corresponds to Godunov, Engquist-Osher and Lax-Friedrichs numerical fluxes as follows: let $\psi \in \operatorname{Lip}\left(I_{0}\right)$ such that

$$
\psi(\theta)=0 \quad \theta \in\left[\bar{B}-\delta_{2}, \bar{A}-\delta_{1}\right]
$$

Let $L^{G}$ denote the Godunov numerical flux associated to $l$. Then the Interface flux $\bar{F}^{G}$ be defined by,

$$
\bar{F}^{G}(a, b)=L^{G}(a, b) \quad a, b \in I_{0}
$$

and the interface flux associated to $\bar{F}^{\psi}$ by

$$
\bar{F}^{\psi}(a, b)=\frac{1}{2}\left[l(a)+l(b)-\int_{a}^{b}\left|\psi^{\prime}(\theta)\right| \mathrm{d} \theta\right] .
$$

By suitable choices of $\psi, \bar{F}^{\psi}$ give raise to Engquist-Osher and Lax-Friedrichs numerical fluxes.

Then we have the following.

Lemma 3.4. Let $\bar{F}^{G}, \bar{F}^{\psi}$ be defined as above. Then

(1) $\bar{F}^{G}$ satisfies conditions (i) and (ii) of the definition of monotone flux (Def. 2.1) and stability condition (2.10). Furthermore it coincides with the interface Godunov flux given in [3] when $A=\theta_{g}$ or $B=\theta_{f}$, that is, in the case of undercompressive, for $a, b \in I$

$$
\bar{F}^{G}(a, b)=\max \left(G^{G}(a, S), F^{G}(s, b)\right)
$$

and in the case of overcompressive, for $a, b \in I$,

$$
\bar{F}^{G}\left(\alpha_{1}(a), \alpha_{2}(b)\right)=\max \left(G^{G}(a, S), F^{G}(s, b)\right)
$$


(2) Let $\psi$ satisfies the following CFL condition

$$
\left\|l^{\prime}\right\|_{\infty} \leq\left\|\psi^{\prime}\right\|_{\infty}
$$

then following holds.

(i) $\bar{F}^{\psi}$ is monotone in the sense of Definition 2.1 and $\bar{F}^{\psi}$ satisfies the stability condition (2.10).

(ii) $\bar{F}^{\psi}$ satisfies the consistency property at the end points, i.e., in the case of undercompressive,

$$
\begin{aligned}
& \bar{F}^{\psi}(s, s)=f(s)=g(s) \\
& \bar{F}^{\psi}(S, S)=f(S)=g(S)
\end{aligned}
$$

and in the case of overcompressive,

$$
\begin{aligned}
& \bar{F}^{\psi}\left(s-\delta_{2}, s-\delta_{2}\right)=\bar{f}\left(s-\delta_{2}\right)=f(s)=g(s) \\
& \bar{F}^{\psi}\left(S-\delta_{1}, S-\delta_{1}\right)=\bar{g}\left(S-\delta_{1}\right)=g(S)=f(S) .
\end{aligned}
$$

Proof. Since $\alpha_{j}$ 's are increasing functions. Therefore $\bar{F}^{G}$ satisfies (i) of Definition 2.1. Now (ii) of Definition 2.1 follows from

$$
\begin{aligned}
\bar{F}^{G}(A, B) & =L^{G}\left(\alpha_{1}(A), \alpha_{2}(B)\right) \\
& =g(A)=F(B) .
\end{aligned}
$$

We verify the result in the case of $A=\theta_{g}$, for other case $A<\theta_{g}$ the result follow similarly. Now

$$
\begin{aligned}
G^{G}(a, S) & =\left\{\begin{array}{lll}
g(a) & \text { if } & a \geq \theta_{g} \\
g\left(\theta_{g}\right) & \text { if } & a \leq \theta_{g}
\end{array}\right. \\
& = \begin{cases}\bar{g}\left(\alpha_{1}(a)\right) & \text { if } \quad \alpha_{1}(a) \geq \alpha_{1}\left(\theta_{g}\right) \\
\bar{g}\left(\alpha_{1}\left(\theta_{g}\right)\right) & \text { if } \quad \alpha_{1}(a) \leq \alpha_{1}\left(\theta_{g}\right) .\end{cases}
\end{aligned}
$$

Similarly $F^{G}(s, b)$ is given by

$$
F^{G}(s, b)=\left\{\begin{array}{lll}
\bar{f}\left(\alpha_{2}(b)\right) & \text { if } & \alpha_{2}(b) \leq \alpha_{2}\left(\theta_{f}\right) \\
\bar{f}\left(\alpha_{2}\left(\theta_{f}\right)\right) & \text { if } & \alpha_{2}(b) \geq \alpha_{2}\left(\theta_{f}\right) .
\end{array}\right.
$$

Now consider several cases,

(1) $b \leq \theta_{f}, a \geq \theta_{g}$, then $\alpha_{2}(b) \leq \alpha_{1}(a)$

$$
\begin{aligned}
\max \left(G^{G}(a, S), F^{G}(s, b)\right) & =\max (g(a), f(b)) \\
& =\max \left(\bar{g}\left(\alpha_{1}(a)\right), \bar{f}\left(\alpha_{2}(b)\right)\right) \\
& =L^{G}\left(\alpha_{1}(a), \alpha_{2}(b)\right) .
\end{aligned}
$$

(2) $b \leq \theta_{f}, a \leq \theta_{g}$, then $\alpha_{2}(b) \leq \alpha_{1}\left(\theta_{g}\right)$

$$
\begin{aligned}
& \max \left(G^{G}(a, S), F^{G}(s, b)\right)=\max \left(g\left(\theta_{g}\right), f(b)\right) \\
& =\left\{\begin{array}{lll}
f(b) & \text { if } & g\left(\theta_{g}\right) \leq f(b) \\
g\left(\theta_{g}\right) & \text { if } & g\left(\theta_{g}\right) \geq f(b)
\end{array}\right. \\
& =\left\{\begin{array}{lll}
\bar{f}\left(\alpha_{2}(b)\right) & \text { if } \quad \alpha_{2}(b) \leq \alpha_{1}(a) \\
\bar{g}\left(\alpha_{1}\left(\theta_{g}\right)\right) & \text { if } \quad \alpha_{2}(b) \geq \alpha_{1}(a) \text { or } \bar{B} \leq \alpha_{1}(b) \leq \alpha_{1}(a) \leq \alpha_{1}\left(\theta_{g}\right)
\end{array}\right. \\
& =L^{G}\left(\alpha_{1}(a), \alpha_{2}(b)\right) \text {. }
\end{aligned}
$$


(3) $b>\theta_{f}, a \leq \theta_{g}$, hence, $\alpha_{2}(b)>\alpha_{2}\left(\theta_{f}\right)$

$$
\max \left(G^{G}(a, S), F^{G}(s, b)\right)=\max \left(g\left(\theta_{g}\right), f\left(\theta_{f}\right)\right)=g\left(\theta_{g}\right),
$$

and if $\alpha_{1}(a) \leq \alpha_{2}(b)$,

$$
L^{G}\left(\alpha_{1}(a), \alpha_{2}(b)\right)=\min _{\left[\alpha_{1}(a), \alpha_{2}(b)\right]} l=l\left(\alpha_{1}\left(\theta_{g}\right)\right)=g\left(\theta_{g}\right),
$$

and if $\alpha_{2}(b) \leq \alpha_{1}(a)$, then $\bar{B} \leq \alpha_{2}(b) \leq \alpha_{1}(a) \leq \alpha_{1}\left(\theta_{g}\right)$,

$$
L^{G}\left(\alpha_{1}(a), \alpha_{2}(b)\right)=\max _{\left[\alpha_{2}(b), \alpha_{1}(a)\right]} l=l\left(\alpha_{2}\left(\theta_{f}\right)\right)=g\left(\theta_{g}\right) .
$$

(4) $b>\theta_{f}, a>\theta_{g}$, then $l\left(\alpha_{1}(a)\right)=g(a), l\left(\alpha_{2}(b)\right)=g\left(\theta_{g}\right)$,

$$
\max \left(G^{G}(a, S), F^{G}(s, b)\right)=\max \left(g(a), f\left(\theta_{f}\right)\right)=g(a),
$$

and if $\alpha_{1}(a) \leq \alpha_{2}(b)$,

$$
L^{G}\left(\alpha_{1}(a), \alpha_{2}(b)\right)=\min _{\left[\alpha_{1}(a), \alpha_{2}(b)\right]} l=l\left(\alpha_{1}(a)\right)=g(a),
$$

and if $\alpha_{1}(a) \geq \alpha_{2}(b)$

$$
L^{G}\left(\alpha_{1}(a), \alpha_{2}(b)\right)=\max _{\left[\alpha_{2}(b), \alpha_{1}(a)\right]} l=l\left(\alpha_{1}(a)\right)=g(a) .
$$

This proves $L^{G}=\bar{F}^{G}$.

Since $\psi=0$ on $[\bar{B}, \bar{A}]$, it follows that

$$
\begin{aligned}
\bar{F}^{\psi}(A, B) & =\frac{1}{2}\left(l\left(\alpha_{1}(A)\right)+l\left(\alpha_{2}(B)\right)-\int_{\alpha_{1}(A)}^{\alpha_{2}(B)}\left|\psi^{\prime}(\theta)\right| \mathrm{d} \theta\right) \\
& \left.=l\left(\alpha_{1}(A)\right)=g(A)=f(B)\right]
\end{aligned}
$$

From (3.10) we have

$$
\begin{aligned}
\frac{\partial \bar{F}^{\psi}}{\partial a}(a, b) & =\frac{1}{2}\left(l^{\prime}\left(\alpha_{1}(a)\right)+\left|\psi^{\prime}\left(\alpha_{1}(a)\right)\right|\right) \geq 0 \\
\frac{\partial \bar{F}}{\partial b}(a, b) & =\frac{1}{2}\left(l^{\prime}\left(\alpha_{2}(b)\right)-\left|\psi^{\prime}\left(\alpha_{2}(b)\right)\right|\right) \leq 0 .
\end{aligned}
$$

Hence $F^{\psi}$ satisfies (i) and (ii) of Definition 2.1. This Proves the lemma.

By taking different choices of $\psi$, we construct interface numerical fluxes corresponds to Engquist-Osher and Lax-Friedrichs scheme as follows:

(i) Engquist-Osher numerical flux. Let $\psi=l$, we have

$$
\bar{F}^{E O}(a, b)=\frac{1}{2}\left(l(a)+l(b)-\int_{a}^{b}\left|l^{\prime}(\theta)\right| \mathrm{d} \theta\right) .
$$

In the case of undercompressive $a, b \in[s, S]$ and in the overcompressive case $a, b \in\left[s-\delta_{2}, S-\delta_{1}\right]$.

(ii) Lax-Friedrichs numerical flux. Here we choose $\psi$ such that $\psi^{\prime}(\theta)=\frac{1}{\lambda} \operatorname{sgn}\left(l^{\prime}(\theta)\right)$, then

$$
\bar{F}^{L F}(a, b)=\frac{1}{2}\left(l(a)+l(b)-\frac{1}{\lambda} \int_{a}^{b}\left|\operatorname{sgn}\left(l^{\prime}(\theta)\right)\right| \mathrm{d} \theta\right) .
$$


(iii) Rusanov or Local Lax-Friedrichs numerical flux. Here we choose $\psi^{\prime}(\theta)=m \operatorname{sgn}\left(l^{\prime}(\theta)\right)$ where $m=$ $\max \left|l^{\prime}(\theta)\right|$ and maximum is taken in between $a$ and $b$.

$$
\bar{F}^{l o c-L F}(a, b)=\frac{1}{2}\left(l(a)+l(b)-m \int_{a}^{b}\left|\operatorname{sgn}\left(l^{\prime}(\theta)\right)\right| \mathrm{d} \theta\right) .
$$

In the undercompressive case $a, b \in I$ and for overcompressive case $a, b \in\left[s-\delta_{2}, S-\delta_{1}\right]$.

Remark 3.5. We remark that a similar analysis to what is done in this paper for $f$ and $g$ satisfying hypothesis $(\mathbf{H})$ can be done for the case where $f$ and $g$ satisfy following hypothesis:

$(\tilde{\mathbf{H}}) . f$ and $g$ have one global maximum no other local maximum in $[s, S]$ and $f, g$ satisfies (i) and (ii) of $(\mathbf{H})$.

Remark 3.6. We have the following explicit formula for the Lax-Friedrichs interface numerical flux. Denote $a \wedge b=\min (a, b)$ and $a \vee b=\max (a, b)$. Then

$$
\bar{F}^{\mathrm{LF}}(a, b)=\frac{1}{2}\left[C(a, b)-\frac{1}{\lambda} D(a, b)\right]
$$

where $C(a, b)$ and $D(a, b)$ given by the following.

(i) Undercompressive case. If $f$ and $g$ satisfies the hypothesis $(\mathbf{H})$, then

$$
\begin{aligned}
& C(a, b)=f(a \wedge \bar{B})+f(b \wedge \bar{B})+g(b \vee \bar{A})+g(a \vee \bar{A})-2 g(A), \\
& D(a, b)=(b \wedge \bar{B})+(b \vee \bar{A})-(a \wedge \bar{B})-(a \vee \bar{A})
\end{aligned}
$$

If $f$ and $g$ satisfies the hypothesis $(\tilde{\mathbf{H}})$, then

$$
\begin{aligned}
& C(a, b)=g(a \wedge \bar{A})+g(b \wedge \bar{A})+f(b \vee \bar{B})+f(a \vee \bar{B})-2 g(A), \\
& D(a, b)=(b \wedge \bar{A})+(b \vee \bar{B})-(a \wedge \bar{A})-(a \vee \bar{B})
\end{aligned}
$$

(ii) Overcompressive case. If $f$ and $g$ satisfies the hypothesis $(\mathbf{H})$, then

$$
\begin{aligned}
C(a, b)= & f\left(\alpha_{1}^{-1}\left(\alpha_{1}^{-1}(a) \wedge \alpha_{2}(\bar{B})\right)\right)+f\left(\alpha_{1}^{-1}\left(\alpha_{2}^{-1}(b) \wedge \alpha_{2}(\bar{B})\right)\right) \\
& +g\left(\alpha_{2}^{-1}\left(\alpha_{1}^{-1}(a) \vee \alpha_{1}(\bar{A})\right)\right)+g\left(\alpha_{2}^{-1}\left(\alpha_{2}^{-1}(b) \vee \alpha_{1}(\bar{A})\right)\right)-2 g(A), \\
D(a, b)= & \left(\alpha_{2}^{-1}(b) \wedge \alpha_{2}(\bar{B})\right)+\left(\alpha_{2}^{-1}(b) \vee \alpha_{1}(\bar{A})\right) \\
& -\left(\alpha_{1}^{-1}(a) \wedge \alpha_{2}(\bar{B})\right)-\left(\alpha_{1}^{-1}(a) \vee \alpha_{1}(\bar{A})\right) .
\end{aligned}
$$

If $f$ and $g$ satisfies the hypothesis $(\tilde{\mathbf{H}})$, then

$$
\begin{aligned}
C(a, b)= & g\left(\alpha_{1}^{-1}\left(\alpha_{1}^{-1}(a) \wedge \alpha_{1}(\bar{A})\right)\right)+g\left(\alpha_{1}^{-1}\left(\alpha_{2}^{-1}(b) \wedge \alpha_{1}(\bar{A})\right)\right) \\
& +f\left(\alpha_{2}^{-1}\left(\alpha_{1}^{-1}(a) \vee \alpha_{2}(\bar{B})\right)\right)+f\left(\alpha_{2}^{-1}\left(\alpha_{2}^{-1}(b) \vee \alpha_{2}(\bar{B})\right)\right)-2 g(A), \\
D(a, b)= & \left(\alpha_{2}^{-1}(b) \wedge \alpha_{1}(\bar{A})\right)+\left(\alpha_{2}^{-1}(b) \vee \alpha_{2}(\bar{B})\right) \\
& -\left(\alpha_{1}^{-1}(a) \wedge \alpha_{1}(\bar{A})\right)-\left(\alpha_{1}^{-1}(a) \vee \alpha_{2}(\bar{B})\right) .
\end{aligned}
$$


Basic motivation to construct $(A, B)$ stable monotone schemes is to take $\bar{F}$ in $(2.7)$ as any one of $\bar{F}^{G}, \bar{F}^{E O}$ or $\bar{F}^{L F}$. Unfortunately if we take $\bar{F}=\bar{F}^{L F}$, then the scheme (2.7) is not monotone as seen below. Assume that $f$ and $g$ has undercompressive intersection. Let $a, b$ be such that $a, b>\bar{A}$ and define

$$
H(a, b, c)=b-\lambda\left[F^{L F}(b, c)-\bar{F}(a, b)\right] .
$$

Then we have $\frac{\partial H}{\partial b}=-\frac{\lambda}{2}\left(f^{\prime}(b)-g^{\prime}(b)\right)$. So we can not determine the sign of $\frac{\partial H}{\partial b}$. But in the case of Godunov and Engquist-Osher, the scheme (2.7) is monotone and $(A, B)$ stable.

Therefore in the case of Lax-Friedrichs, scheme (2.7) has to be modified so that it is monotone and $(A, B)$ stable. This is done by modifying (2.7) at $i=2$ and $i=-1$. First we define for the undercompressive case. Using the translation invariance property of solutions, we can define the similar schemes for the other cases.

Godunov, Engquist-Osher and Lax-Friedrichs schemes. Now we define the different numerical schemes corresponds to $\bar{F}^{G}, \bar{F}^{E O}, \bar{F}^{L F}, \bar{F}^{l o c-L F}$. Define $\tilde{F}$ and $\tilde{G}$ as follows.

$$
\begin{gathered}
\tilde{F}(a, b)= \begin{cases}F^{G}(a, b) & \text { if } \bar{F}=\bar{F}^{G} \\
F^{E O}(a, b) & \text { if } \bar{F}=\bar{F}^{E O} \\
\frac{1}{2}\left(l(a)+f(b)-\frac{b-a}{\lambda}\right) & \text { if } \bar{F}=\bar{F}^{L F} \\
\frac{1}{2}\left(l(a)+f(b)-m_{1}(b-a)\right) & \text { if } \bar{F}=\bar{F}^{l o c-L F}\end{cases} \\
\tilde{G}(a, b)= \begin{cases}G^{G}(a, b) & \text { if } \bar{F}=\bar{F}^{G} \\
G^{E O}(a, b) & \text { if } \bar{F}=\bar{F}^{E O} \\
\frac{1}{2}\left(g(a)+l(b)-\frac{b-a}{\lambda}\right) & \text { if } \bar{F}=\bar{F}^{L F} \\
\frac{1}{2}\left(g(a)+l(b)-m_{2}(b-a)\right) & \text { if } \bar{F}=\bar{F}^{l o c-L F}\end{cases}
\end{gathered}
$$

where $m_{1}$ is the maximum of $\left|f^{\prime}\right|$ and $\left|l^{\prime}\right|$ over the interval $[a, b]$ or $[b, a]$ and $m_{2}$ is the maximum of $\left|g^{\prime}\right|$ and $\left|l^{\prime}\right|$ over the interval $[a, b]$ or $[b, a]$. Next we define the scheme as follows

$$
\begin{aligned}
& u_{i}^{n+1}=u_{i}^{n}-\lambda\left(F\left(u_{i}^{n}, u_{i+1}^{n}\right)-F\left(u_{i-1}^{n}, u_{i}^{n}\right)\right) \\
& u_{2}^{n+1}=u_{2}^{n}-\lambda\left(F\left(u_{2}^{n}, u_{3}^{n}\right)-\tilde{F}\left(u_{1}^{n}, u_{2}^{n}\right)\right) \\
& u_{1}^{n+1}=u_{1}^{n}-\lambda\left(\tilde{F}\left(u_{1}^{n}, u_{2}^{n}\right)-\bar{F}\left(u_{0}^{n}, u_{1}^{n}\right)\right) \\
& u_{0}^{n+1}=u_{0}^{n}-\lambda\left(\bar{F}\left(u_{0}^{n}, u_{1}^{n}\right)-\tilde{G}\left(u_{-1}^{n}, u_{0}^{n}\right)\right) \\
& u_{-1}^{n+1}=u_{-1}^{n}-\lambda\left(\tilde{G}\left(u_{-1}^{n}, u_{0}^{n}\right)-G\left(u_{-2}^{n}, u_{-1}^{n}\right)\right) \\
& u_{i}^{n+1}=u_{i}^{n}-\lambda\left(G\left(u_{i}^{n}, u_{i+1}^{n}\right)-G\left(u_{i-1}^{n}, u_{i}^{n}\right)\right)
\end{aligned}
$$

Convergence of numerical schemes. It is well known that for the discontinuous flux, Godunov and Engquist-Osher schemes are monotone. It was an open problem to derive a monotone numerical scheme like Lax-Friedrichs scheme which produces $(A, B)$ entropy solution for the scalar conservation laws with discontinuous flux. Here we first show that Lax-Friedrichs scheme defined above is monotone. In the next section, using the singular mapping and chain estimates we prove the theorems. In the end, we give some numerical experiments to show the performance of the above Lax-Friedrichs scheme. 
For the simplicity, we will concentrate only on the undercompressive case of fluxes $f$ and $g$. For the other cases the convergence analysis follows similarly. To prove the convergence we first show that the above scheme is monotone. To see this, define the following:

$$
\begin{aligned}
H_{3}(X, Y, Z) & =Y-\lambda(F(Y, Z)-F(X, Y)) \\
H_{2}(X, Y, Z) & =Y-\lambda(F(Y, Z)-\tilde{F}(X, Y)) \\
H_{1}(X, Y, Z) & =Y-\lambda(\tilde{F}(Y, Z)-\bar{F}(X, Y)) \\
H_{-1} & =Y-\lambda(\bar{F}(Y, Z)-\tilde{G}(X, Y)) \\
H_{-2} & =Y-\lambda(\tilde{G}(Y, Z)-G(X, Y)) \\
H_{-3} & =Y-\lambda(G(Y, Z)-G(X, Y)) .
\end{aligned}
$$

Next we have the following lemma.

Lemma 3.7. Let $2 \lambda M \leq 1$ and $a, b, c \in[s, S]$, then

(i) $H_{i}(s, s, s)=f(s)=g(s)$ and $H_{i}(S, S, S)=f(S)=g(S)$.

(ii) $\quad a \mapsto \tilde{F}(a, b), a \mapsto \tilde{G}(a, b)$ are non decreasing and $b \mapsto \tilde{F}(a, b), b \mapsto \tilde{G}(a, b)$ are non increasing.

(iii) $H_{i}$ is non decreasing in each of its variables.

(iv) $H_{i}$ satisfies the following relations:

$$
\begin{aligned}
& \frac{\partial H_{3}}{\partial X}(a, \cdot, \cdot)+\frac{\partial H_{3}}{\partial Y}(\cdot, a, \cdot)+\frac{\partial H_{3}}{\partial Z}(\cdot, \cdot, a)=1 \\
& \frac{\partial H_{3}}{\partial X}(a, \cdot, \cdot)+\frac{\partial H_{3}}{\partial Y}(\cdot, a, \cdot)+\frac{\partial H_{2}}{\partial Z}(\cdot, \cdot, a)=1 \\
& \frac{\partial H_{3}}{\partial X}(a, \cdot, \cdot)+\frac{\partial H_{2}}{\partial Y}(\cdot, a, \cdot)+\frac{\partial H_{1}}{\partial Z}(\cdot, \cdot, a)=1 \\
& \frac{\partial H_{2}}{\partial X}(a, \cdot, \cdot)+\frac{\partial H_{1}}{\partial Y}(\cdot, a, \cdot)+\frac{\partial H_{-1}}{\partial Z}(\cdot, \cdot, a)=1 \\
& \frac{\partial H_{1}}{\partial X}(a, \cdot, \cdot)+\frac{\partial H_{-1}}{\partial Y}(\cdot, a, \cdot)+\frac{\partial H_{-2}}{\partial Z}(\cdot, \cdot, a)=1 \\
& \frac{\partial H_{-1}}{\partial X}(a, \cdot, \cdot)+\frac{\partial H_{-2}}{\partial Y}(\cdot, a, \cdot)+\frac{\partial H_{-3}}{\partial Z}(\cdot, \cdot, a)=1 \\
& \frac{\partial H_{-2}}{\partial X}(a, \cdot, \cdot)+\frac{\partial H_{-3}}{\partial Y}(\cdot, a, \cdot)+\frac{\partial H_{-3}}{\partial Z}(\cdot, \cdot, a)=1 \\
& \frac{\partial H_{-3}}{\partial X}(a, \cdot, \cdot)+\frac{\partial H_{-3}}{\partial Y}(\cdot, a, \cdot)+\frac{\partial H_{-3}}{\partial Z}(\cdot, \cdot, a)=1 .
\end{aligned}
$$

Proof. (i) directly follows from the explicit formula of numerical fluxes. Now from the definition of $\tilde{F}$, we have

$$
\tilde{F}(a, b)=\frac{l(a)+f(b)}{2}-\frac{1}{2 \lambda}(b-a) .
$$

Therefore, under the CFL condition we obtain

$$
\begin{aligned}
& \frac{\partial \tilde{F}}{\partial a}=\frac{l^{\prime}(a)}{2}+\frac{1}{2 \lambda} \geq 0 \\
& \frac{\partial \tilde{F}}{\partial b}=\frac{l^{\prime}(b)}{2}-\frac{1}{2 \lambda}=\frac{1}{2 \lambda}\left(\lambda f^{\prime}(b)-1\right) \geq 0 .
\end{aligned}
$$


This shows that $a \mapsto \tilde{F}(a, b)$ is non decreasing and $b \mapsto \tilde{F}(a, b)$ is non increasing. Similarly we can also prove for $\tilde{G}$. This concludes the proof of (ii).

From the monotonocity of the numerical fluxes it is clear that $X \mapsto H_{i}(X, Y, Z)$ and $Z \mapsto H_{i}(X, Y, Z)$ are non decreasing. So we only need to show that $Y \mapsto H_{i}(X, Y, Z)$ is non decreasing. Now using the explicit formula of numerical fluxes we obtain

$$
\begin{aligned}
\frac{\partial H_{3}}{\partial Y} & =1-\lambda\left(\frac{\partial F}{\partial a}(Y, Z)-\frac{\partial F}{\partial b}(x, Y)\right)=1-\lambda\left(\frac{f^{\prime}(Y)}{2}+\frac{1}{2 \lambda}\right)+\lambda\left(\frac{f^{\prime}(Y)}{2}-\frac{1}{2 \lambda}\right) \\
& =0 \\
\frac{\partial H_{2}}{\partial Y} & =1-\lambda\left(\frac{\partial F}{\partial a}(Y, Z)-\frac{\partial \tilde{F}}{\partial b}(x, Y)\right)=1-\lambda\left(\frac{f^{\prime}(Y)}{2}+\frac{1}{2 \lambda}\right)+\lambda\left(\frac{f^{\prime}(Y)}{2}-\frac{1}{2 \lambda}\right) \\
& =0, \\
\frac{\partial H_{1}}{\partial Y}=1 & -\lambda\left(\frac{\partial \tilde{F}}{\partial a}(Y, Z)-\frac{\partial \bar{F}}{\partial b}(x, Y)\right)=1-\lambda\left(\frac{l^{\prime}(Y)}{2}+\frac{1}{2 \lambda}\right)+\lambda\left(\frac{l^{\prime}(Y)}{2}-\frac{1}{2 \lambda}\left|\operatorname{sgn}\left(l^{\prime}(b)\right)\right|\right) \\
=\frac{1}{2} & \left(1-\left|\operatorname{sgn}\left(l^{\prime}(b)\right)\right|\right) \geq 0 .
\end{aligned}
$$

This shows that $Y \mapsto H_{i}(X, Y, Z), i=1,2,3$ are non decreasing. Similarly we can also prove that $Y \mapsto$ $H_{i}(X, Y, Z), i=-1,-2,-3$ are non decreasing. This concludes the proof of (iii).

Now,

$$
\begin{aligned}
\frac{\partial H_{3}}{\partial X}(a, \cdot, \cdot)+\frac{\partial H_{3}}{\partial Y}(\cdot, a, \cdot)+\frac{\partial H_{3}}{\partial Z}(\cdot, \cdot, a)= & \lambda \frac{\partial F}{\partial a}(a, \cdot)+1-\lambda\left(\frac{\partial F}{\partial a}(a, \cdot)-\frac{\partial F}{\partial b}(\cdot, a)\right) \\
& -\lambda \frac{\partial F}{\partial b}(\cdot, a)=1, \\
\frac{\partial H_{3}}{\partial X}(a, \cdot, \cdot)+\frac{\partial H_{3}}{\partial Y}(\cdot, a, \cdot)+\frac{\partial H_{2}}{\partial Z}(\cdot, \cdot, a)= & \lambda \frac{\partial F}{\partial a}(a, \cdot)+1-\lambda\left(\frac{\partial F}{\partial a}(a, \cdot)-\frac{\partial F}{\partial b}(\cdot, a)\right) \\
& -\lambda \frac{\partial F}{\partial b}(\cdot, a)=1, \\
\frac{\partial H_{3}}{\partial X}(a, \cdot, \cdot)+\frac{\partial H_{2}}{\partial Y}(\cdot, a, \cdot)+\frac{\partial H_{1}}{\partial Z}(\cdot, \cdot, a)= & \lambda \frac{\partial F}{\partial a}(a, \cdot)+1-\lambda\left(\frac{\partial F}{\partial a}(a, \cdot)-\frac{\partial \tilde{F}}{\partial b}(\cdot, a)\right) \\
& -\lambda \frac{\partial \tilde{F}}{\partial b}(\cdot, a)=1, \\
\frac{\partial H_{2}}{\partial X}(a, \cdot, \cdot)+\frac{\partial H_{1}}{\partial Y}(\cdot, a, \cdot)+\frac{\partial H_{-1}}{\partial Z}(\cdot, \cdot, a)= & \lambda \frac{\partial \tilde{F}}{\partial a}(a, \cdot)+1-\lambda\left(\frac{\partial \tilde{F}}{\partial a}(a, \cdot)-\frac{\partial \bar{F}}{\partial b}(\cdot, a)\right) \\
& -\lambda \frac{\partial \bar{F}}{\partial b}(\cdot, a)=1, \\
\frac{\partial H_{1}}{\partial X}(a, \cdot, \cdot)+\frac{\partial H_{-1}}{\partial Y}(\cdot, a, \cdot)+\frac{\partial H_{-2}}{\partial Z}(\cdot, \cdot, a)= & \lambda \frac{\partial \bar{F}}{\partial a}(a, \cdot)+1-\lambda\left(\frac{\partial \bar{F}}{\partial a}(a, \cdot)-\frac{\partial \tilde{F}}{\partial b}(\cdot, a)\right) \\
& -\lambda \frac{\partial \tilde{G}}{\partial b}(\cdot, a)=1 .
\end{aligned}
$$


This proves the first, second, third, fourth and fifth equality of (iv). Other equalities follows similarly. This completes the proof of (iv).

\section{Proof of Theorems 2.1 And 2.2}

The aim of this section is to prove Theorems 2.1 and 2.2 for the scheme (3.16).

To prove the theorems, we need the following lemma which gives $L^{\infty}$-bounds and local $L^{1}$-contraction of the scheme (2.7). The idea of the proof is taken from [18].

\section{Lemma 4.1.}

(i) Let $u_{0} \in L^{\infty}(\mathbb{R},[s, S])$ be the initial data and $u_{i}^{n}$ be the solution satisfying (2.7). Then

$$
s \leq u_{i}^{n} \leq S \quad \text { for all } i, n .
$$

(ii) Let $u_{0}, v_{0} \in L^{\infty}(\mathbb{R},[s, S])$ be two initial data, and $\left\{u_{i}^{n}\right\},\left\{v_{i}^{n}\right\}$ be the corresponding solution of (2.7) respectively. Then we have the following

$$
\begin{gathered}
\sum_{i_{0} \leq i \leq j_{0}}\left|u_{i}^{n+1}-v_{i}^{n+1}\right| \leq \sum_{i_{0}-1 \leq i \leq j_{0}+1}\left|u_{i}^{n}-v_{i}^{n}\right| \\
\sum_{i}\left|u_{i}^{n+1}-v_{i}^{n+1}\right| \leq \sum_{i}\left|u_{i}^{n}-v_{i}^{n}\right|
\end{gathered}
$$

Proof. Proof of this lemma for the case of Godunov and Engquist-Osher schemes can be found in [5,9]. Therefore we let $F=F^{L F}, G=G^{L F}, \bar{F}=\bar{F}^{L F}$ in (3.16). Since $s \leq u_{0} \leq S$ and hence for all $i, s \leq u_{i}^{0} \leq S$. By induction, assume that (4.1) holds for all $n$. Then from (i) and (ii) of Lemma 3.2 we have

$$
\begin{aligned}
& s=H_{3}(s, s, s) \leq H_{3}\left(u_{i-1}^{n}, u_{i}^{n}, u_{i+1}^{n}\right)=u_{i}^{n+1} \leq H_{3}(S, S, S)=S \quad \text { if } \quad i \geq 3 \\
& s=H_{2}(s, s, s) \leq H_{2}\left(u_{1}^{n}, u_{2}^{n}, u_{3}^{n}\right)=u_{2}^{n+1} \leq H_{2}(S, S, S)=S \\
& s=H_{1}(s, s, s) \leq H_{1}\left(u_{0}^{n}, u_{1}^{n}, u_{2}^{n}\right)=u_{1}^{n+1} \leq H_{1}(S, S, S)=S \\
& s=H_{-1}(s, s, s) \leq H_{-1}\left(u_{-1}^{n}, u_{0}^{n}, u_{1}^{n}\right)=u_{0}^{n+1} \leq H_{-1}(S, S, S)=S \\
& s=H_{-2}(s, s, s) \leq H_{-2}\left(u_{-2}^{n}, u_{-1}^{n}, u_{0}^{n}\right)=u_{-1}^{n+1} \leq H_{-2}(S, S, S)=S \\
& s=H_{-3}(s, s, s) \leq H_{-3}\left(u_{i-1}^{n}, u_{i}^{n}, u_{i+1}^{n}\right)=u_{i}^{n+1} \leq H_{-3}(S, S, S)=S \quad \text { if } \quad i \leq-2 .
\end{aligned}
$$

This proves (4.1). As the scheme (2.7) is monotone and conservative, the second inequality of (ii) follows as an application of Crandall-Tartar Lemma. Next we prove the first inequality of (ii). We will prove this inequality for $i_{0} \leq 0$ and $j_{0} \geq 1$. The other cases follow in the same manner. For $\theta \in[0,1]$, we define $p_{i}^{n}(\theta)=\theta u_{i}^{n}+(1-\theta) v_{i}^{n}$. As $\frac{\partial H_{i}}{\partial X}(X, Y, Z)$ is independent of $Y$ and $Z, \frac{\partial H_{i}}{\partial Y}(X, Y, Z)$ is independent of $X$ and $Z$ and $\frac{\partial H_{i}}{\partial Z}(X, Y, Z)$ is independent of $X$ and $Y$, henceforth we use the following notation

$$
\frac{\partial H_{i}}{\partial X}(X, Y, Z)=: \frac{\partial H_{i}}{\partial X}(X), \quad \frac{\partial H_{i}}{\partial Y}(X, Y, Z)=: \frac{\partial H_{i}}{\partial Y}(Y), \quad \frac{\partial H_{i}}{\partial Z}(X, Y, Z)=: \frac{\partial H_{i}}{\partial Z}(Z) .
$$


Now using the mean value theorem, we obtain

$$
\begin{aligned}
& \sum_{i=3}^{j_{0}}\left|u_{i}^{n+1}-v_{i}^{n+1}\right| \\
& =\sum_{i=3}^{j_{0}}\left|H_{3}\left(u_{i-1}^{n}, u_{i}^{n}, u_{i+1}^{n}\right)-H_{3}\left(v_{i-1}^{n}, v_{i}^{n}, v_{i+1}^{n}\right)\right| \\
& \leq \sum_{i=3}^{j_{0}}\left(\left|u_{i-1}^{n}-v_{i-1}^{n}\right| \int_{0}^{1} \frac{\partial H_{3}}{\partial X}\left(p_{i-1}^{n}(\theta)\right) \mathrm{d} \theta+\left|u_{i}^{n}-v_{i}^{n}\right| \int_{0}^{1} \frac{\partial H_{3}}{\partial Y}\left(p_{i}^{n}(\theta)\right) \mathrm{d} \theta\right. \\
& \left.+\left|u_{i+1}^{n}-v_{i+1}^{n}\right| \int_{0}^{1} \frac{\partial H_{3}}{\partial Z}\left(p_{i+1}^{n}(\theta)\right) \mathrm{d} \theta\right) \\
& =\left|u_{2}^{n}-v_{2}^{n}\right| \int_{0}^{1} \frac{\partial H_{3}}{\partial X}\left(p_{2}^{n}(\theta)\right) \mathrm{d} \theta+\left|u_{3}^{n}-v_{3}^{n}\right| \int_{0}^{1}\left(\frac{\partial H_{3}}{\partial X}\left(p_{3}^{n}(\theta)\right)+\frac{\partial H_{3}}{\partial Y}\left(p_{3}^{n}(\theta)\right)\right) \mathrm{d} \theta \\
& +\sum_{i=4}^{j_{0}-1}\left|u_{i}^{n}-v_{i}^{n}\right|\left(\frac{\partial H_{3}}{\partial X}\left(p_{i}^{n}(\theta)\right)+\frac{\partial H_{3}}{\partial Y}\left(p_{i}^{n}(\theta)\right)+\frac{\partial H_{3}}{\partial Z}\left(p_{i}^{n}(\theta)\right)\right) \mathrm{d} \theta \\
& +\left|u_{j_{0}}^{n}-v_{j_{0}}^{n}\right| \int_{0}^{1}\left(\frac{\partial H_{3}}{\partial Y}\left(p_{j_{0}}^{n}(\theta)\right)+\frac{\partial H_{3}}{\partial Z}\left(p_{j_{0}}^{n}(\theta)\right)\right) \mathrm{d} \theta \\
& +\left|u_{j_{0}+1}^{n}-v_{j_{0}+1}^{n}\right| \int_{0}^{1} \frac{\partial H_{3}}{\partial Z}\left(p_{j_{0}+1}^{n}(\theta)\right) \mathrm{d} \theta \\
& \left|u_{2}^{n+1}-v_{2}^{n+1}\right|+\left|u_{1}^{n+1}-v_{1}^{n+1}\right| \\
& =\left|H_{2}\left(u_{1}^{n}, u_{2}^{n}, u_{3}^{n}\right)-H_{2}\left(v_{1}^{n}, v_{2}^{n}, v_{3}^{n}\right)\right|+\left|H_{1}\left(u_{0}^{n}, u_{1}^{n}, u_{2}^{n}\right)-H_{1}\left(v_{0}^{n}, v_{1}^{n}, v_{2}^{n}\right)\right| \\
& \leq\left|u_{0}^{n}-v_{0}^{n}\right| \int_{0}^{1} \frac{\partial H_{1}}{\partial X}\left(p_{0}^{n}(\theta)\right) \mathrm{d} \theta+\left|u_{1}^{n}-v_{1}^{n}\right| \int_{0}^{1}\left(\frac{\partial H_{2}}{\partial X}\left(p_{1}^{n}(\theta)\right)+\frac{\partial H_{1}}{\partial Y}\left(p_{1}^{n}(\theta)\right)\right) \mathrm{d} \theta \\
& +\left|u_{2}^{n}-v_{2}^{n}\right| \int_{0}^{1}\left(\frac{\partial H_{2}}{\partial Y}\left(p_{2}^{n}(\theta)\right)+\frac{\partial H_{1}}{\partial Z}\left(p_{2}^{n}(\theta)\right)\right) \mathrm{d} \theta+\left|u_{3}^{n}-v_{3}^{n}\right| \int_{0}^{1} \frac{\partial H_{2}}{\partial Z}\left(p_{3}^{n}(\theta)\right) \mathrm{d} \theta \\
& \left|u_{0}^{n+1}-v_{0}^{n+1}\right| \leq\left|u_{-1}^{n}-v_{-1}^{n}\right| \int_{0}^{1} \frac{\partial H_{-1}}{\partial X}\left(p_{-1}^{n}(\theta)\right) \mathrm{d} \theta+\left|u_{0}^{n}-v_{0}^{n}\right| \int_{0}^{1} \frac{\partial H_{-1}}{\partial Y}\left(p_{0}^{n}(\theta)\right) \mathrm{d} \theta \\
& +\left|u_{1}^{n}-v_{1}^{n}\right| \int_{0}^{1} \frac{\partial H_{-1}}{\partial Z}\left(p_{1}^{n}(\theta)\right) \mathrm{d} \theta, \\
& \left|u_{-2}^{n+1}-v_{-2}^{n+1}\right|+\left|u_{-1}^{n+1}-v_{-1}^{n+1}\right| \\
& \leq\left|u_{0}^{n}-v_{0}^{n}\right| \int_{0}^{1} \frac{\partial H_{-2}}{\partial Z}\left(p_{0}^{n}(\theta)\right) \mathrm{d} \theta+\left|u_{-1}^{n}-v_{-1}^{n}\right| \int_{0}^{1}\left(\frac{\partial H_{-2}}{\partial Y}\left(p_{-1}^{n}(\theta)\right)+\frac{\partial H_{-3}}{\partial Z}\left(p_{-1}^{n}(\theta)\right)\right) \mathrm{d} \theta \\
& +\left|u_{-2}^{n}-v_{-2}^{n}\right| \int_{0}^{1}\left(\frac{\partial H_{-2}}{\partial X}\left(p_{-2}^{n}(\theta)\right)+\frac{\partial H_{-3}}{\partial X}\left(p_{-3}^{n}(\theta)\right)\right) \mathrm{d} \theta \\
& +\left|u_{-3}^{n}-v_{-3}^{n}\right| \int_{0}^{1} \frac{\partial H_{-3}}{\partial X}\left(p_{-3}^{n}(\theta)\right) \mathrm{d} \theta,
\end{aligned}
$$


and

$$
\begin{aligned}
& \sum_{i_{0}}^{-2}\left|u_{i}^{n+1}-v_{i}^{n+1}\right| \\
= & \sum_{i_{0}}^{-2}\left|H_{-3}\left(u_{i-1}^{n}, u_{i}^{n}, u_{i+1}^{n}\right)-H_{-3}\left(v_{i-1}^{n}, v_{i}^{n}, v_{i+1}^{n}\right)\right| \\
\leq & \left|u_{-1}^{n}-v_{-1}^{n}\right| \int_{0}^{1} \frac{\partial H_{-3}}{\partial Z}\left(p_{-1}^{n}(\theta)\right) \mathrm{d} \theta+\left|u_{-2}^{n}-v_{-2}^{n}\right| \int_{0}^{1}\left(\frac{\partial H_{-3}}{\partial Y}\left(p_{-2}^{n}(\theta)\right)+\frac{\partial H_{-3}}{\partial Z}\left(p_{-2}^{n}(\theta)\right)\right) \mathrm{d} \theta \\
& +\sum_{i=i_{0}+1}^{-3}\left|u_{i}^{n}-v_{i}^{n}\right| \int_{0}^{1}\left(\frac{\partial H_{-3}}{\partial x}\left(p_{i}^{n}(\theta)\right)+\frac{\partial H_{-3}}{\partial Y}\left(p_{i}^{n}(\theta)\right)+\frac{\partial H_{-3}}{\partial Z}\left(p_{i}^{n}(\theta)\right)\right) \\
& +\left|u_{i_{0}}^{n}-v_{i_{0}}^{n}\right| \int_{0}^{1}\left(\frac{\partial H_{-3}}{\partial x}\left(p_{i_{0}}^{n}(\theta)\right)+\frac{\partial H_{-3}}{\partial Y}\left(p_{i_{0}}^{n}(\theta)\right)\right) \\
& +\left|u_{i_{0}-1}^{n}-v_{i_{0}-1}^{n}\right| \int_{0}^{1} \frac{\partial H_{-3}}{\partial x}\left(p_{i_{0}-1}^{n}(\theta)\right) \mathrm{d} \theta .
\end{aligned}
$$

Summing up all the above inequalities and using equalities (iii) of Lemma 3.2 we obtain the result. This proves the lemma.

Following [3], next we introduce singular mapping and we prove that the solution of the scheme (2.7) has a total variation bound with respect to the singular mapping. This is done via the chain estimate of Adimurthi and Gowda ([19], p. 209).

\subsection{Singular mapping and chain estimates}

Let $k \in \operatorname{Lip}(I)$ such that $k$ is not locally constant in a neighbourhood of a point of interior local maxima if it exist. Define $k\left(\theta_{k}\right)=\min _{I} k$. Let $a, b \in \mathbb{R}$ and $u \in I$, define $a_{+}=\max (a, 0), a_{-}=\min (a, 0)$ so $a=a_{+}+a_{-}$, $|a|=a_{+}-a_{-}$. In addition, we define

$$
\begin{gathered}
\chi(a, b)=\left\{\begin{array}{lll}
1 & \text { if } & a<b, \\
0 & \text { if } & a \geq b,
\end{array}\right. \\
\chi_{-}(k, u)=\left\{\begin{array}{lll}
1 & \text { if } & u \leq \theta_{k}, \\
0 & \text { if } & u>\theta_{k},
\end{array}\right. \\
\left.\chi_{+}(k, u)\right)=\left\{\begin{array}{lll}
1 & \text { if } & u>\theta_{k}, \\
0 & \text { if } & u \leq \theta_{k} .
\end{array}\right.
\end{gathered}
$$

Let $K$ be a monotone numerical flux associated to $k$. Then we have the following results.

Proposition 4.2. Let $a, b \in I$, then

$$
\begin{gathered}
K(a, b)-k\left(\theta_{k}\right) \leq 0 \quad \text { if } \quad a \leq \theta_{k} \leq b, \\
k(b)-K(a, b) \leq 0 \quad \text { if } \quad b \leq a .
\end{gathered}
$$

Proof. From (i) of Definition 2.1, we have if $a \leq \theta_{k} \leq b$, then

$$
K(a, b)-k\left(\theta_{k}\right) \leq K\left(\theta_{k}, b\right)-k\left(\theta_{k}\right) \leq K\left(\theta_{k}, \theta_{k}\right)-k\left(\theta_{k}\right)=0 .
$$

If $b \leq a$, then

$$
K(a, b)-k(b) \geq K(b, b)-k(b)=0 .
$$

This proves the proposition. 
Lemma 4.3. Let $\left\{v_{1}, \ldots, v_{N}\right\}$ be a sequence in $I$ such that $v_{2} \leq v_{3} \leq \ldots \leq v_{N-1}$. Then (1) If $v_{2} \leq v_{1}, v_{N} \leq v_{N-1}$ then

$$
\int_{v_{2}}^{v_{N-1}}\left|k^{\prime}(\theta)\right| \mathrm{d} \theta \leq \sum_{j=2}^{N-1}\left|K\left(v_{j}, v_{j+1}\right)-K\left(v_{j-1}, v_{j}\right)\right| .
$$

(2) If $v_{N} \leq v_{N-1}$ then

$$
\begin{aligned}
\int_{v_{1}}^{v_{N-1}}\left|k^{\prime}(\theta)\right| \mathrm{d} \theta \leq \sum_{j=2}^{N-1} \mid & K\left(v_{j}, v_{j+1}\right)-K\left(v_{j-1}, v_{j}\right) \mid \\
& +\chi\left(v_{1}, v_{2}\right)\left(k\left(v_{1}\right)-K\left(v_{1}, v_{2}\right)\right)\left(\chi_{-}\left(k, v_{1}\right)-\chi_{+}\left(k, v_{2}\right)\right) .
\end{aligned}
$$

(3) If $v_{2} \leq v_{1}$ then

$$
\begin{aligned}
\int_{v_{2}}^{v_{N}}\left|k^{\prime}(\theta)\right| \mathrm{d} \theta \leq & \sum_{j=2}^{N-1}\left|K\left(v_{j}, v_{j+1}\right)-K\left(v_{j-1}, v_{j}\right)\right| \\
& +\chi\left(v_{N}, v_{N-1}\right)\left(k\left(v_{N}\right)-K\left(v_{N-1}, v_{N}\right)\right)\left(\chi_{+}\left(k, v_{N}\right)-\chi_{-}\left(k, v_{N-1}\right)\right) .
\end{aligned}
$$

Proof.

(1) Let $i$ be such that $v_{i-1} \leq \theta_{k} \leq v_{i}$. Since $v_{N} \leq v_{N-1}, v_{2} \leq v_{1}$ then from (4.5) and (4.6)

$$
\begin{aligned}
& k\left(v_{N}\right)-K\left(v_{N-1}, v_{N}\right) \leq 0, \\
& k\left(v_{2}\right)-K\left(v_{1}, v_{2}\right) \leq 0, \\
& K\left(v_{i-1}, v_{i}\right)-k\left(\theta_{k}\right) \leq 0 .
\end{aligned}
$$

Hence

$$
\begin{aligned}
\int_{v_{2}}^{v_{N-1}} k_{+}^{\prime}(\theta) \mathrm{d} \theta & =\int_{\theta_{k}}^{v_{N-1}} k^{\prime}(\theta) \mathrm{d} \theta \\
& =k\left(v_{N-1}\right)-k\left(\theta_{k}\right) \\
& =\left(k\left(v_{N-1}\right)-K\left(v_{N-1}, v_{N}\right)\right)+\sum_{j=i}^{N-1}\left(K\left(v_{j}, v_{j+1}\right)-K\left(v_{j-1}, v_{j}\right)\right)+\left(K\left(v_{i-1}, v_{i}\right)-k\left(\theta_{k}\right)\right) \\
& \leq \sum_{j=i}^{N-1}\left|K\left(v_{j}, v_{j+1}\right)-K\left(v_{j-1}, v_{j}\right)\right| .
\end{aligned}
$$

and

$$
\begin{aligned}
-\int_{v_{2}}^{v_{N-1}} k_{-}^{\prime}(\theta) \mathrm{d} \theta & =-\int_{v_{2}}^{\theta_{k}} k^{\prime}(\theta) \mathrm{d} \theta \\
& =k\left(v_{2}\right)-k\left(\theta_{k}\right) \\
& =\left(k\left(v_{2}\right)-K\left(v_{1}, v_{2}\right)\right)-\sum_{j=2}^{i-1}\left(K\left(v_{j}, v_{j+1}\right)-K\left(v_{j-1}, v_{j}\right)\right)+\left(K\left(v_{i-1}, v_{i}\right)-k\left(\theta_{k}\right)\right) \\
& \leq \sum_{j=2}^{i-1}\left|K\left(v_{j}, v_{j+1}\right)-K\left(v_{j-1}, v_{j}\right)\right| .
\end{aligned}
$$

Now (4.7) follows adding the above two estimates. 
(2) Let $v_{N} \leq v_{N-1}$. If $v_{2} \leq v_{1}$, then $\chi\left(v_{1}, v_{2}\right)\left(k\left(v_{1}\right)-K\left(v_{1}, v_{2}\right)\right)=0$ and hence (4.8) follows from (4.7). Hence assume that $v_{1}<v_{2}$. Let $v_{1} \geq \theta_{k}$, then from (4.6)

$$
\begin{aligned}
\int_{v_{1}}^{v_{N-1}}\left|k^{\prime}(\theta)\right| \mathrm{d} \theta & =k\left(v_{N-1}\right)-k\left(v_{1}\right) \\
& =\left(k\left(v_{N-1}\right)-K\left(v_{N-1}, v_{N}\right)\right)+\sum_{j=2}^{N-1}\left(K\left(v_{j}, v_{j+1}\right)-K\left(v_{j-1}, v_{j}\right)\right)+\left(K\left(v_{1}, v_{2}\right)-k\left(v_{1}\right)\right) \\
& \leq \sum_{j=2}^{N-1}\left|K\left(v_{j}, v_{j+1}\right)-K\left(v_{j-1}, v_{j}\right)\right|+\left(K\left(v_{1}, v_{2}\right)-k\left(v_{1}\right)\right) .
\end{aligned}
$$

If $v_{1} \leq \theta_{k}$, then choose $i$ such that $v_{i-1} \leq \theta_{k} \leq v_{i}$ and processed as in (1) to obtain

$$
\int_{v_{1}}^{v_{N-1}}\left|k^{\prime}(\theta)\right| \mathrm{d} \theta \leq\left(k\left(v_{1}\right)-K\left(v_{1}, v_{2}\right)\right)+\sum_{j=2}^{N-1}\left|K\left(v_{j}, v_{j+1}\right)-K\left(v_{j-1}, v_{j}\right)\right| .
$$

Now (4.8) follows from these two estimates.

(3) Let $v_{2} \leq v_{1}$. If $v_{N} \leq v_{N-1}$, then $\chi\left(v_{N-1}, v_{N}\right)\left(k\left(v_{N}\right)-K\left(v_{N-1}, v_{N}\right)\right)=0$, hence (4.9) follows from (4.7). Therefore assume $v_{N-1}<v_{N}$. Suppose $v_{N} \leq \theta_{k}$, then

$$
\begin{aligned}
\int_{v_{2}}^{v_{N}}\left|k^{\prime}(\theta)\right| \mathrm{d} \theta & =k\left(v_{2}\right)-k\left(v_{N}\right) \\
& =\left(k\left(v_{2}\right)-K\left(v_{1}, v_{2}\right)\right)+\sum_{j=2}^{N-1}\left(K\left(v_{j}, v_{j+1}\right)-K\left(v_{j-1}, v_{j}\right)\right)+\left(K\left(v_{N-1}, v_{N}\right)-k\left(v_{N}\right)\right) \\
& \leq \sum_{j=2}^{N-1}\left|K\left(v_{j}, v_{j+1}\right)-K\left(v_{j-1}, v_{j}\right)\right|+\left(K\left(v_{N-1}, v_{N}\right)-k\left(v_{N}\right)\right)
\end{aligned}
$$

If $v_{N}>\theta_{k}$, choose $i$ such that $v_{i-1} \leq \theta_{k} \leq v_{i}$ and proceed as in (1) to obtain

$$
\int_{v_{2}}^{v_{N}}\left|k^{\prime}(\theta)\right| \mathrm{d} \theta \leq\left(k\left(v_{N}\right)-K\left(v_{N-1}, v_{N}\right)\right)+\sum_{j=2}^{N-1}\left|K\left(v_{j}, v_{j+1}\right)-K\left(v_{j-1}, v_{j}\right)\right| .
$$

Now (4.9) follows from these two estimates. This proves the lemma.

Definition 4.4. Let $k \in \operatorname{Lip}(I)$ and $A \in I$, define the singular mapping $\psi_{k, A}$ associated to $k$ and $A$ by

$$
\psi_{k, A}(u)=\int_{A}^{u}\left|k^{\prime}(\theta)\right| \mathrm{d} \theta
$$

\subsection{Total variation bounds with respect to singular mapping}

Let $k_{1}, k_{2} \in \operatorname{Lip}(I)$ satisfying the same hypothesis as in Proposition 4.1. Let $A_{1}, A_{2} \in I$ and define

$$
\psi_{1}=\psi_{k_{1}, A_{1}} \quad \psi_{2}=\psi_{k_{2}, A_{2}}
$$

Let $K_{1}$ and $K_{2}$ be two monotone numerical flux associated to $k_{1}$ and $k_{2}$ respectively. Let $\left\{v_{i}\right\} \subset I$ be a sequence. Define the new sequence $\left\{V_{i}\right\}$ by

$$
V_{i}= \begin{cases}\psi_{1}\left(v_{i}\right) & \text { if } \quad i \geq 1 \\ \psi_{2}\left(v_{i}\right) & \text { if } i \quad \leq 0\end{cases}
$$


Let $\eta_{-}=\varlimsup_{i \rightarrow-\infty} V_{i}$ and $\eta_{+}=\underline{\lim }_{i \rightarrow \infty} V_{i}, \eta=\eta_{-} \eta_{+}$. Then we have the following inequalities

$$
\begin{aligned}
\sum_{i=-\infty}^{\infty}\left(V_{i}-V_{i+1}\right) & =\lim _{N_{1} \rightarrow \infty, N_{2} \rightarrow-\infty} \sum_{N_{2}}^{N_{1}}\left(V_{i}-V_{i+1}\right) \\
& =\lim _{N_{1} \rightarrow \infty, N_{2} \rightarrow-\infty}\left(V_{N_{2}}-V_{N_{1}+1}\right) \\
& \leq \varlimsup_{N \rightarrow-\infty} V_{N}-\varliminf_{N \rightarrow \infty} V_{N}=\eta_{-}-\eta_{+}=\eta
\end{aligned}
$$

Hence

$$
\sum_{i=-\infty}^{\infty}\left(V_{i}-V_{i+1}\right)_{+} \leq-\sum_{i=-\infty}^{\infty}\left(V_{i}-V_{i+1}\right)_{-}+\eta
$$

Therefore,

$$
\begin{aligned}
\operatorname{TV}\left(\left\{V_{i}\right\}\right)=\sum_{i=-\infty}^{\infty}\left|V_{i}-V_{i+1}\right| & =\sum_{i=-\infty}^{\infty}\left(V_{i}-V_{i+1}\right)_{+}-\sum_{i=-\infty}^{\infty}\left(V_{i}-V_{i+1}\right)_{-} \\
& \leq-2 \sum_{i=-\infty}^{\infty}\left(V_{i}-V_{i+1}\right)_{-}+\eta
\end{aligned}
$$

Next we decompose the sequence $\left\{v_{i}\right\}$ into oscillatory and non-oscillatory parts as follows: define the subsets $\left\{J_{i}\right\}$ having the following properties. $\left\{v_{i}\right\}=\cup J_{i}$. Let $J_{1}=\left\{v_{1}, v_{2}, \ldots, v_{i_{1}-1}\right\}$ such that

$$
\left\{\begin{array}{l}
v_{2} \leq v_{3} \leq \ldots \leq v_{i_{1}-1} \\
v_{i_{1}}<v_{i_{1}-1}
\end{array}\right.
$$

For $l \geq 1$, define $J_{l+1}=\left\{v_{i_{l}}, v_{i_{l}+1}, \ldots, v_{i_{l+1}-1}\right\}$,

$$
\left\{\begin{array}{l}
v_{i_{l}} \leq v_{i_{l}+1} \leq \ldots \leq v_{i_{l+1}-1} \\
v_{i_{l+1}}<v_{i_{l+1}-1}
\end{array}\right.
$$

$J_{0}=\left\{v_{i_{0}+1}, \ldots, v_{0}\right\}$

$$
\left\{\begin{array}{l}
v_{i_{0}+1} \leq \ldots \leq v_{-2} \leq v_{-1} \\
v_{i_{0}+1}<v_{i_{0}}
\end{array}\right.
$$

For $l \leq-1$ define $J_{l}=\left\{v_{i_{l}+1}, \ldots, v_{i_{l-1}}\right\}$

$$
\left\{\begin{array}{l}
v_{i_{l}+1} \leq \ldots \leq v_{i_{l+1}} \\
v_{i_{l}+1}<v_{i_{l}} .
\end{array}\right.
$$

Since $\psi_{1}, \psi_{2}$ are non decreasing functions and hence from (4.7) to (4.9) we have

$$
\begin{array}{lll}
\left(\psi_{2}\left(v_{i_{j}}\right)-\psi_{2}\left(v_{i_{j}+1}\right)\right)_{-}=0 & \text { for } & j \leq 0 \\
\left(\psi_{1}\left(v_{i_{j}-1}\right)-\psi_{1}\left(v_{i_{j}}\right)\right)_{-}=0 & \text { for } & j \geq 1
\end{array}
$$


So, using Lemma 4.2 , we have

$$
\begin{aligned}
& -\left(\psi_{2}\left(v_{-1}\right)-\psi_{2}\left(v_{0}\right)\right)_{-}-\sum_{i=i_{0}+1}^{-2}\left(\psi_{2}\left(v_{i}\right)-\psi_{2}\left(v_{i+1}\right)\right)_{-} \\
& =-\left(\psi_{2}\left(v_{-1}\right)-\psi_{2}\left(v_{0}\right)\right)_{-}+\psi_{2}\left(v_{-1}\right)-\psi_{2}\left(v_{i_{0}+1}\right) \\
& =\left\{\begin{array}{l}
\int_{v_{i_{0}+1}}^{v_{0}}\left|k_{2}^{\prime}(\theta)\right| \mathrm{d} \theta \quad \text { if } v_{-1} \leq v_{0} \\
\int_{v_{i_{0}+1}}^{v_{-1}}\left|k_{2}^{\prime}(\theta)\right| \mathrm{d} \theta \quad \text { if } v_{-1} \geq v_{0} \\
\leq \chi\left(v_{-1}, v_{0}\right)\left(k_{2}\left(v_{0}\right)-K_{2}\left(v_{-1}, v_{0}\right)\right)\left(\chi_{+}\left(k_{2}, v_{0}\right)-\chi_{-}\left(k_{2}, v_{0}\right)\right) \\
+\sum_{j=i_{0}+1}^{-1}\left|K_{2}\left(v_{j}, v_{j+1}\right)-K_{2}\left(v_{j-1}, v_{j}\right)\right| .
\end{array}\right.
\end{aligned}
$$

Similarly, using Lemma 4.2, we have

$$
\begin{gathered}
-\left(\psi_{1}\left(v_{1}\right)-\psi_{1}\left(v_{2}\right)\right)_{-}-\sum_{i=2}^{i_{1}-1}\left(\psi_{1}\left(v_{i}\right)-\psi_{1}\left(v_{i+1}\right)\right)_{-} \\
\leq \chi\left(v_{1}, v_{2}\right)\left(k_{1}\left(v_{1}\right)-K_{1}\left(v_{1}, v_{2}\right)\right)\left(\chi_{-}\left(k_{1}, v_{1}\right)-\chi_{+}\left(k_{1}, v_{1}\right)\right) \\
+\sum_{j=2}^{i_{1}-1}\left|K_{1}\left(v_{j}, v_{j+1}\right)-K_{1}\left(v_{j-1}, v_{j}\right)\right| . \\
\sum_{v_{i} \in J_{i}}\left(\psi_{2}\left(v_{i}\right)-\psi_{2}\left(v_{i+1}\right)\right)_{-} \leq \sum_{v_{i} \in J_{j}}\left|K_{2}\left(v_{i}, v_{i+1}\right)-K_{2}\left(v_{i-1}, v_{i}\right)\right| \quad \text { for } j \leq-1 . \\
\sum_{v_{i} \in J_{i}}\left(\psi_{1}\left(v_{i}\right)-\psi_{1}\left(v_{i+1}\right)\right)_{-} \leq \sum_{v_{i} \in J_{j}}\left|K_{1}\left(v_{i}, v_{i+1}\right)-K_{1}\left(v_{i-1}, v_{i}\right)\right| \quad \text { for } j \geq 2 .
\end{gathered}
$$

Combining all the estimates from (4.20) to (4.23) obtain the following Lemma.

Lemma 4.5 (Chain estimate). Let $\left\{v_{i}\right\}$ and $\left\{V_{i}\right\}$ be the sequence defined as above, then

$$
\begin{aligned}
\frac{1}{2} T V\left(\left\{V_{i}\right\}\right) \leq & -\left(\psi_{2}\left(v_{0}\right)-\psi_{1}\left(v_{1}\right)\right)_{-}+\sum_{i=-\infty}^{-1}\left|K_{2}\left(v_{i}, v_{i+1}\right)-K_{2}\left(v_{i-1}, v_{i}\right)\right| \\
& +\sum_{i=2}^{\infty}\left|K_{1}\left(v_{i}, v_{i+1}\right)-K_{1}\left(v_{i-1}, v_{i}\right)\right|+\frac{\eta}{2} \\
& +\chi\left(v_{1}, v_{2}\right)\left(k_{1}\left(v_{1}\right)-K_{1}\left(v_{1}, v_{2}\right)\right)\left(\chi_{-}\left(k_{1}, v_{1}\right)-\chi_{+}\left(k_{1}, v_{1}\right)\right) \\
& +\chi\left(v_{-1}, v_{0}\right)\left(k_{2}\left(v_{0}\right)-K_{2}\left(v_{-1}, v_{0}\right)\right)\left(\chi_{+}\left(k_{2}, v_{0}\right)-\chi_{-}\left(k_{2}, v_{0}\right)\right) .
\end{aligned}
$$

With these preliminaries, let us go back to the fluxes $f$ and $g$. Let $F$ and $G$ be two monotone fluxes associated to $f$ and $g$ respectively. Let $\bar{F}$ satisfies condition (i) of Definition 2.1. We choose $k_{1}=f, k_{2}=g$ and define,

$$
z_{i}^{n}=\left\{\begin{array}{lll}
\psi_{1}\left(u_{i}^{n}\right) & \text { if } & i \geq 1 \\
\psi_{2}\left(u_{i}^{n}\right) & \text { if } \quad i \leq 0 .
\end{array}\right.
$$


We define,

$$
\begin{aligned}
E= & \left.-\left(z_{0}^{n}-z_{1}^{n}\right)_{-}+\chi\left(u_{1}^{n}, u_{2}^{n}\right)\left(f\left(u_{1}^{n}\right)-F\left(u_{1}^{n}, u_{2}^{n}\right)\right)\left(\chi_{-}\left(f, u_{1}^{n}\right)-\chi_{+}\left(f, u_{1}^{n}\right)\right)\right) \\
& +\chi\left(u_{-1}^{n}, u_{0}^{n}\right)\left(g\left(u_{0}^{n}\right)-G\left(u_{-1}^{n}, u_{0}^{n}\right)\right)\left(\chi_{+}\left(g, u_{0}^{n}\right)-\chi_{-}\left(g, u_{0}^{n}\right)\right) \\
& -\left|\bar{F}\left(u_{0}^{n}, u_{1}^{n}\right)-\tilde{G}\left(u_{-1}^{n}, u_{0}^{n}\right)\right|-\left|\tilde{F}\left(u_{1}^{n}, u_{2}^{n}\right)-\bar{F}\left(u_{0}^{n}, u_{1}^{n}\right)\right| \\
& -\left|F\left(u_{2}^{n}, u_{3}^{n}\right)-\tilde{F}\left(u_{1}^{n}, u_{2}^{n}\right)\right|-\left|\tilde{G}\left(u_{-1}^{n}, u_{0}^{n}\right)-G\left(u_{-2}^{n}, u_{-1}^{n}\right)\right| .
\end{aligned}
$$

Lemma 4.6. Let $z_{i}^{n}$ be as defined above, then the following estimate holds, for all $m, n \in \mathbb{N}$,

$$
\begin{aligned}
T V\left(z_{i}^{n}\right) & \leq 2 S T V\left(u_{0}\right)+2 E+\eta \\
\sum_{i}\left|z_{i}^{n}-z_{i}^{m}\right| & \leq \lambda L|n-m| S T V\left(u_{0}\right)
\end{aligned}
$$

where $E$ is given by (4.25) and $S T V\left(u_{0}\right)$ is given by (2.8) and

$$
L=\max \left\{\operatorname{Lip}\left(\psi_{1}\right), \operatorname{Lip}\left(\psi_{2}\right)\right\} .
$$

Proof. Using the chain estimate (Lem. 4.3) and Lemma 4.1 we have,

$$
\begin{aligned}
\sum_{-\infty}^{\infty}\left|z_{i}^{n}-z_{i-1}^{n}\right| \leq & 2\left(\sum_{i=-\infty}^{i=-2}\left|G\left(u_{i}^{n}, u_{i+1}^{n}\right)-G\left(u_{i-1}^{n}, u_{i}^{n}\right)\right|\right. \\
& +\left|\tilde{G}\left(u_{-1}^{n}, u_{0}^{n}\right)-\bar{F}\left(u_{0}^{n}, u_{1}^{n}\right)\right|+\left|\tilde{F}\left(u_{1}^{n}, u_{2}^{n}\right)-\bar{F}\left(u_{0}^{n}, u_{1}^{n}\right)\right| \\
& +\left|\tilde{G}\left(u_{-1}^{n}, u_{0}^{n}\right)-G\left(u_{-2}^{n}, u_{-1}^{n}\right)\right|+\mid \tilde{F}\left(u_{1}^{n}, u_{2}^{n}\right)-F\left(u_{2}^{n}, u_{3}^{n}\right) \\
& \left.+\sum_{i=3}^{\infty}\left|F\left(u_{i}^{n}, u_{i+1}^{n}\right)-F\left(u_{i-1}^{n}, u_{i}^{n}\right)\right|\right)+E+\eta \\
\leq & \frac{2}{\lambda} \sum_{-\infty}^{\infty}\left|u_{i}^{n+1}-u_{i}^{n}\right|+2 E+\eta \\
\leq & \frac{2}{\lambda} \sum_{-\infty}^{\infty}\left|u_{i}^{1}-u_{i}^{0}\right|+2 E+\eta \\
\leq & 2 \operatorname{STV}\left(u_{0}\right)+2 E+\eta .
\end{aligned}
$$

It is easy to see that for each $n, E$ is bounded independent of the mesh parameters. To prove the second inequality, without loss of generality we assume that $n \geq m$. Then from Lemma 4.1 we have,

$$
\begin{aligned}
\sum_{i}\left|z_{i}^{n}-z_{i}^{m}\right| & =\sum_{i \leq 0}\left|z_{i}^{n}-z_{i}^{m}\right|+\sum_{i \geq 1}\left|z_{i}^{n}-z_{i}^{m}\right| \\
& \leq L \sum_{i}\left|u_{i}^{n}-u_{i}^{m}\right| \\
& \leq L \sum_{i} \sum_{j=0}^{n-m+1}\left|u_{i}^{n-j}-u_{i}^{n-j-1}\right| \\
& \leq L|n-m| \sum_{i}\left|u_{i}^{1}-u_{i}^{0}\right| \\
& \leq \lambda L|n-m| \operatorname{STV}\left(u_{0}\right) .
\end{aligned}
$$

This completes the proof. 
Next we show that if the monotone scheme converges to the solution then the solution satisfies interface entropy condition. To prove that we use the idea of Crandall and Majda [10].

Numerical interface entropy condition. Here following the idea of Crandall and Majda [10] we show that the monotone scheme for (1.2) satisfy the interface entropy condition. For $a, b \in \mathbb{R}$, let $a \vee b=\max (a, b), a \wedge b=$ $\min (a, b)$. Let $l_{1}, l_{2} \in I$, define

$$
\begin{aligned}
& \tilde{F}^{e}(a, b)=\tilde{F}\left(a \vee l_{2}, b \vee l_{2}\right)-\tilde{F}\left(a \wedge l_{2}, b \wedge l_{2}\right), \\
& \tilde{G}^{e}(a, b)=\tilde{G}\left(a \vee l_{1}, b \vee l_{1}\right)-\tilde{G}\left(a \wedge l_{1}, b \wedge l_{1}\right), \\
& \bar{F}^{e}(a, b)=\bar{F}\left(a \vee l_{1}, b \vee l_{2}\right)-\bar{F}\left(a \wedge l_{1}, b \wedge l_{2}\right) .
\end{aligned}
$$

For $\phi \in \mathrm{C}_{0}^{1}\left(\mathbb{R}_{+}\right)$define

$$
\phi_{n}(t)=\phi(n \triangle t), \quad \text { if }(n-1) \triangle t<t \leq n \triangle t .
$$

Then we have the following.

Lemma 4.7. Let $\left\{u_{i}^{n}\right\}$ be the solution of the scheme (2.7) and $u_{h}$ be the corresponding piecewise constant function as in (2.9). Let $l_{1}=A, l_{2}=B, T>0$ and $\phi \in C_{0}^{1}\left(\mathbb{R}_{+}\right)$such that $\phi \geq 0$. Then

$$
\begin{aligned}
& \lambda \int_{0}^{T}\left\{\tilde{G}^{e}\left(u_{h}(-2 h, t), u_{h}(-h, t)\right)-\tilde{F}^{e}\left(u_{h}(h, t), u_{h}(2 h, t)\right)\right\} \phi_{h}(t) \mathrm{d} t \\
& \geq \int_{\triangle t}^{T}\left(\left|u_{h}(-h, t)-A\right|+\left|u_{h}(h, t)-B\right|\right)\left(\phi_{h}(t-\lambda h)-\phi_{h}(t)\right) \mathrm{d} t \\
& +\left(\left|u_{h}(-h, t+\triangle t)-A\right|+\left|u_{h}(h, T+\triangle t)-B\right|\right) \phi_{h}(T) \triangle t \\
& \quad-\left(\left|u_{h}(-h, \triangle t)-A\right|+\left|u_{h}(h, \triangle t)-B\right|\right) \phi_{h}(\triangle t) \triangle t .
\end{aligned}
$$

Proof. Let $a_{1}, a_{2}, a_{3}, a_{4} \in I$, since $H_{ \pm 1}(X, Y, Z)$ is non decreasing function in each of its variable, we have

$$
\begin{aligned}
& \lambda\left(\tilde{G}^{e}\left(a_{1}, a_{2}\right)-\bar{F}^{e}\left(a_{2}, a_{3}\right)\right) \\
= & \lambda\left(\tilde{G}\left(a_{1} \vee l_{1}, a_{2} \vee l_{1}\right)-\bar{F}\left(a_{2} \vee l_{1}, a_{3} \vee l_{2}\right)\right)+a_{2} \vee l_{1}-a_{2} \vee l_{1} \\
& -\lambda\left(\tilde{G}\left(a_{1} \wedge l_{1}, a_{2} \wedge l_{1}\right)-\bar{F}\left(a_{2} \wedge l_{1}, a_{3} \wedge l_{2}\right)\right)+a_{2} \wedge l_{1}-a_{2} \wedge l_{1} \\
= & H_{-1}\left(a_{1} \vee l_{1}, a_{2} \vee l_{1}, a_{3} \vee l_{2}\right)-H_{-1}\left(a_{1} \wedge l_{1}, a_{2} \wedge l_{1}, a_{3} \wedge l_{2}\right)-\left|a_{2}-l_{1}\right| \\
\geq & H_{-1}\left(a_{1}, a_{2}, a_{3}\right) \vee H_{-1}\left(l_{1}, l_{1}, l_{2}\right)-H_{-1}\left(a_{1}, a_{2}, a_{3}\right) \wedge H_{-1}\left(l_{1}, l_{1}, l_{2}\right)-\left|a_{2}-l_{1}\right| \\
= & \left|H_{-1}\left(a_{1}, a_{2}, a_{3}\right)-H_{-1}\left(l_{1}, l_{1}, l_{2}\right)\right|-\left|a_{2}-l_{1}\right|
\end{aligned}
$$

similarly,

$$
\lambda\left(\bar{F}^{e}\left(a_{2}, a_{3}\right)-\tilde{F}^{e}\left(a_{3}, a_{4}\right)\right) \geq\left|H_{1}\left(a_{2}, a_{3}, a_{4}\right)-H_{1}\left(l_{1}, l_{2}, l_{2}\right)\right|-\left|a_{3}-l_{2}\right| .
$$

Adding the above two inequalities we obtain,

$$
\begin{aligned}
\lambda\left(\tilde{G}^{e}\left(a_{1}, a_{2}\right)-\tilde{F}^{e}\left(a_{3}, a_{4}\right)\right) \geq & \left|H_{-1}\left(a_{1}, a_{2}, a_{3}\right)-H_{-1}\left(l_{1}, l_{1}, l_{2}\right)\right|-\left|a_{2}-l_{1}\right| \\
& +\left|H_{1}\left(a_{2}, a_{3}, a_{4}\right)-H_{1}\left(l_{1}, l_{2}, l_{2}\right)\right|-\left|a_{3}-l_{2}\right| .
\end{aligned}
$$


Let $l_{1}=A, l_{2}=B$, then from $(2.10)$ we have $\bar{F}(A, B)=g(A)=f(B)$. Hence

$$
\begin{gathered}
H_{1}(A, B, B)=B-\lambda(\tilde{F}(B, B)-\bar{F}(A, B))=B \\
H_{-1}(A, A, B)=A-\lambda(\bar{F}(A, B)-\tilde{G}(A, A))=A .
\end{gathered}
$$

Now, in (4.30) we choose, $\left(a_{1}, a_{2}, a_{3}, a_{4}\right)=\left(u_{-1}^{n}, u_{0}^{n}, u_{1}^{n}, u_{2}^{n}\right)$ and $\left(l_{1}, l_{2}\right)=(A, B)$ to obtain

$$
\begin{aligned}
\lambda\left(\tilde{G}^{e}\left(u_{-1}^{n}, u_{0}^{n}\right)-\tilde{F}^{e}\left(u_{1}^{n}, u_{2}^{n}\right)\right) \geq & \left|u_{0}^{n+1}-A\right|-\left|u_{0}^{n}-A\right| \\
& +\left|u_{1}^{n+1}-B\right|-\left|u_{1}^{n}-B\right| .
\end{aligned}
$$

Now multiply this by $\phi(n \triangle t)=\phi\left(t_{n}\right)$ and summing from 1 to $N$, (where $n \triangle t=T$ ), we get

$$
\begin{aligned}
\lambda \sum_{n=1}^{N}\left(\tilde{G}^{e}\left(u_{-1}^{n}, u_{0}^{n}\right)-\tilde{F}^{e}\left(u_{1}^{n}, u_{2}^{n}\right)\right) \phi\left(t_{n}\right) \geq & \sum_{2}^{N}\left(\left|u_{0}^{n}-A\right|+\left|u_{1}^{n}-B\right|\right)\left(\phi\left(t_{n-1}-\phi\left(t_{n}\right)\right)\right) \\
& +\left(\left|u_{0}^{N+1}-A\right|+\left|u_{1}^{N+1}-B\right|\right) \phi\left(t_{N}\right) \\
& -\left(\left|u_{0}^{1}-A\right|+\left|u_{1}^{1}-B\right|\right) \phi\left(t_{1}\right) .
\end{aligned}
$$

Multiply by $\triangle t$, we obtain

$$
\begin{aligned}
& \lambda \int_{0}^{T}\left\{\tilde{G}^{e}\left(u_{h}(-2 h, t), u_{h}(-h, t)\right)-\tilde{F}^{e}\left(u_{h}(h, t), u_{h}(2 h, t)\right)\right\} \phi_{h}(t) \mathrm{d} t \\
& \geq \int_{\triangle t}^{T}\left(\left|u_{h}(-h, t)-A\right|+\left|u_{h}(h, t)-B\right|\right)\left(\phi_{h}(t-\lambda h)-\phi_{h}(t)\right) \mathrm{d} t \\
& +\left(\left|u_{h}(-h, t+\triangle t)-A\right|+\left|u_{h}(h, T+\triangle t)-B\right|\right) \phi_{h}(T) \triangle t \\
& -\left(\left|u_{h}(-h, \triangle t)-A\right|+\left|u_{h}(h, \triangle t)-B\right|\right) \phi_{h}(\triangle t) \triangle t
\end{aligned}
$$

This proves the Lemma.

As an immediate consequence of this is that the solution satisfies $(A, B)$-interface entropy condition under suitable assumption on the convergence of the sequence $u_{h}$.

Lemma 4.8. Let for a subsequence $h_{k}, u_{h_{k}} \rightarrow u$ in $L_{l o c}^{\infty}\left(\mathbb{R}_{+}, L_{l o c}^{1}(\mathbb{R})\right)$. Assume also that for almost all $t$, as $h_{k} \rightarrow 0$

$$
\begin{gathered}
\left(u_{h_{k}}\left(-h_{k}, t\right), u_{h_{k}}\left(-2 h_{k}, t\right)\right) \rightarrow(u(0-, t), u(0-, t)) \\
\left(u_{h_{k}}\left(h_{k}, t\right), u_{h_{k}}\left(2 h_{k}, t\right)\right) \rightarrow(u(0+, t), u(0+, t))
\end{gathered}
$$

then $u$ satisfies the interface entropy condition.

Proof. Let $\phi \in \mathrm{C}_{0}^{1}\left(\mathbb{R}_{+}\right)$such that $\phi \geq 0$ and let $T>0$. Since $\phi_{h_{k}}\left(t-h_{k}\right)-\phi_{h_{k}}(t) \rightarrow 0$ a.e. $t$ as $h_{k} \rightarrow 0$, hence from (4.31), (4.32) and by dominated convergence theorem, from Lemma 4.6 we obtain,

$$
\lambda \int_{0}^{T}\left(\tilde{G}^{e}(u(0-, t), u(0-, t))-\tilde{F}^{e}(u(0+, t), u(0+, t))\right) \phi(t) \mathrm{d} t \geq 0 .
$$

Hence for a.e. $t$

$$
\tilde{G}^{e}(u(0-, t), u(0-, t))-\tilde{F}^{e}(u(0+, t), u(0+, t)) \geq 0 .
$$


This implies, for a.e. $t$

$$
\begin{aligned}
& \operatorname{sgn}(u(0-, t)-A)((g(u(0-, t))-g(A))+(l(u(0-, t))-l(A))) \\
& -\operatorname{sgn}(u(0+, t)-B)((f(u(0+, t)-f(B)))+(l(u(0-, t))-l(B))) \geq 0 .
\end{aligned}
$$

The next lemma shows that the $u$ satisfies interface entropy condition. This completes the proof of the Lemma.

Let $u$ be a weak solution such that traces $u(0+, t)$ and $u(0-, t)$ exists for a.e. $t$. Given a connection $(A, B)$, we define the interface entropy functional by

$$
\begin{aligned}
I_{A B}(u)= & \operatorname{sgn}(u(0-, t)-A)((g(u(0-, t))-g(A))+(l(u(0-, t))-l(A))) \\
& -\operatorname{sgn}(u(0+, t)-B)((f(u(0+, t)-f(B)))+(l(u(0+, t))-l(B))) .
\end{aligned}
$$

Lemma 4.9. Let $u$ be a weak solution such that traces $u(0+, t)$ and $u(0-, t)$ exists for a.e. $t$. Assume that $I_{A B}(u) \geq 0$ for a.e. $t$. Then $u$ satisfies the interface entropy condition (2.5).

Proof. We prove the above result for the case of undercompressive fluxes. The other cases will follow similarly. Let $u(0+, t) \geq \bar{B}, u(0-, t) \leq \bar{A}$. Since $f(u(0+, t))=g(u(0-, t))$, hence either $A \leq u(0-, t) \leq \bar{A}$ and $\bar{B} \leq$ $u(0+, t) \leq B$ or $u(0-, t) \leq A$ and $u^{+} \geq B$. We have the following cases.

Case 1. If $u(0-, t)<A$ and $u(0+, t)>B$, then we have

$$
0 \leq I_{A B}(u)=-(g(u(0-, t))-g(A))-(l(u(0-, t))-l(A))-(f(u(0+, t))-f(B))-(l(u(0+, t))-l(B))<0 .
$$

This gives a contradiction.

Case 2. If $A<u(0-, t)<\bar{A}$ and $\bar{B}<u(0+, t)<B$, then we have

$$
0 \leq I_{A B}(u)=(g(u(0-, t))-g(A))+(l(u(0-, t))-l(A))+(f(u(0+, t))-f(B))+(l(u(0+, t))-l(B))<0 .
$$

This gives a contradiction. Therefore $(u(0+, t), u(0-, t))$ is one of the points $(A, B),(\bar{A}, B),(A, \bar{B}),(\bar{A}, \bar{B})$. Hence $u$ satisfies interface entropy condition (2.5). This completes the proof of the lemma.

Next we indicate the proof of Theorems 2.1 and 2.2.

Proof of Theorem 2.1 and Theorem 2.2: The proof is exactly same as in [3]. From Lemma $4.4\left\{z_{i}^{n}\right\}$ are total variation bounded and hence as in [3] there exist a subsequence $h_{k} \rightarrow 0$ such that $u_{h_{k}}$ converges in $\mathrm{L}_{\text {loc }}^{\infty}\left(\mathbb{R}_{+}, \mathrm{L}_{\text {loc }}^{1}(\mathbb{R})\right)$ to a weak solution $u$ of $(1.2)$ with the property that $u(0 \pm, t)$ exist for a.e. $t$ and satisfying Kruzkov entropy condition away from $x=0$. As the solution satisfies interface entropy condition, the proof of Theorem 2.2 is exactly same as in $[3,5]$.

\section{NumERICAL EXPERIMENTS}

In this section, we present some numerical experiments for the Godunov as well as Lax-Friedrichs schemes developed in Section 3 and compare their numerical performances. As in the case $f=g$, in the discontinuous flux case also Lax-Friedrichs scheme is more diffusive compared to Godunov, but when mesh sizes goes to zero both agree. Here we study their numerical performances when $f$ and $g$ have undercompressive as well as overcompressive intersections. In Experiment 1, we consider undercompressive intersection of $f$ and $g$ with undercompressive as well as overcompressive initial data. In Experiment 2, we consider overcompressive intersection of $f$ and $g$ with undercompressive as well as overcompressive initial data. In all the experiments, observe that numerical solution satisfies $(A, B)$ interface entropy condition. We will refer the solution of Godunov scheme 


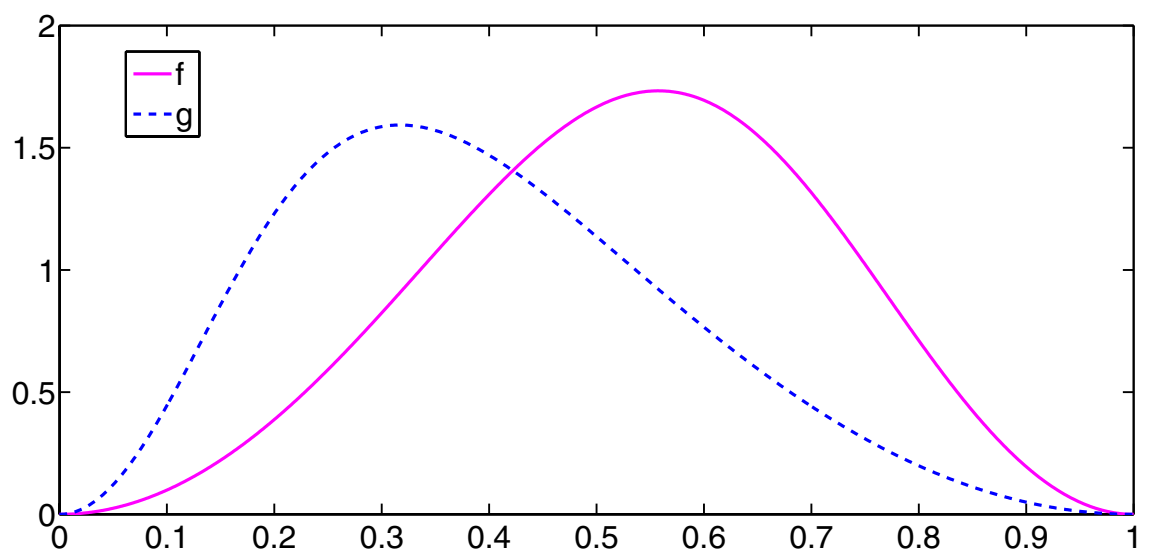

FigURE 6. Undercompressive intersection.

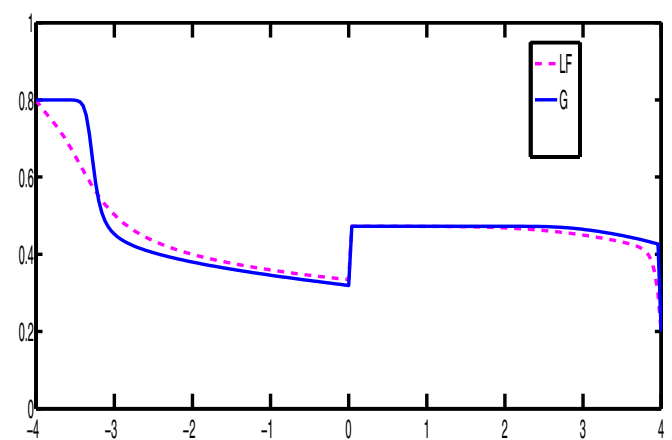

(a)

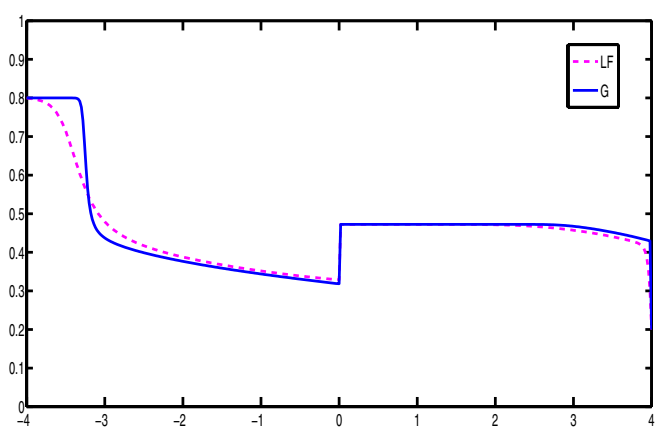

(b)

Figure 7. Solution of Experiment 1.1 at $T=1$.

and Lax-Friedrichs scheme, by $\mathbf{G}$ and $\mathbf{L F}$ respectively. To see the convergence, in all the numerical experiments we compute the solution on coarser as well as on finer mesh.

Experiment 1. Undercompressive intersection case. Here we take the following example of two phase flow as in [3], where $f$ and $g$ are of concave type functions (see Fig. 6) given by

$$
f(u)=\frac{20 u^{2}(1-u)^{2}}{u^{2}+2(1-u)^{2}}, \quad g(u)=\frac{50 u^{2}(1-u)^{2}}{10 u^{2}+(1-u)^{2}} .
$$

We take the connection as $\left(\theta_{g}, B\right)=(0.317014,0.472372)$. With this choice of $f, g$ and connection $\left(\theta_{g}, B\right)$, we compute the solution for the following Riemann problems.

Experiment 1.1. Undercompressive initial data. We take the following undercompressive data,

$$
u_{0}=\left\{\begin{array}{lll}
0.8 & \text { if } \quad x<0 \\
0.2 & \text { if } \quad x>0
\end{array}\right.
$$

that is $f^{\prime}(.2)>0$ and $g^{\prime}(.8)<0$. In Figure 7a, comparison is made between Godunov scheme, Lax-Friedrichs scheme with $\lambda=1 / 16, h=\frac{8}{200}$ and time $T=1$. As expected Lax-Friedrichs scheme more diffusive than Godunov scheme but as mesh size goes to zero Figure $7 \mathrm{~b}\left(\lambda=1 / 16, h=\frac{8}{400}\right)$ shows both agrees. 


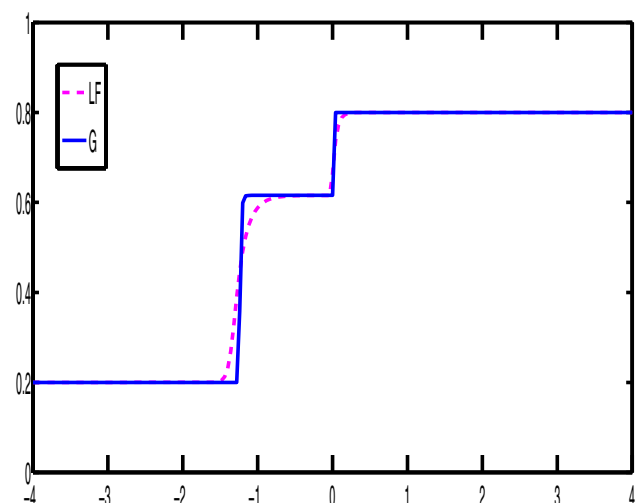

(a)

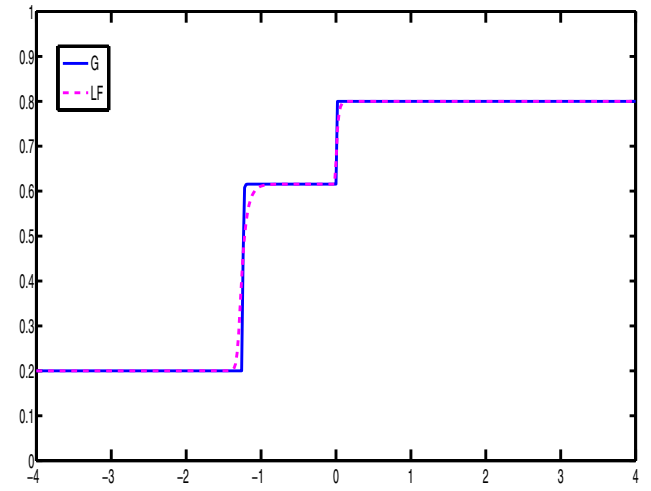

(b)

FiguRE 8. Solution of Experiment 1.2 at $T=1$.

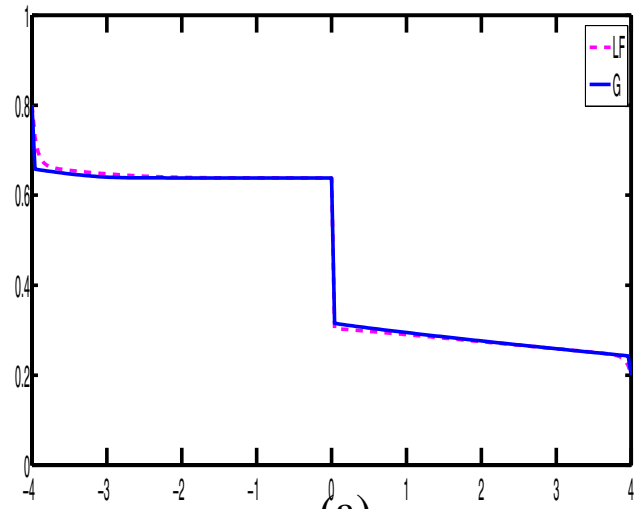

(a)

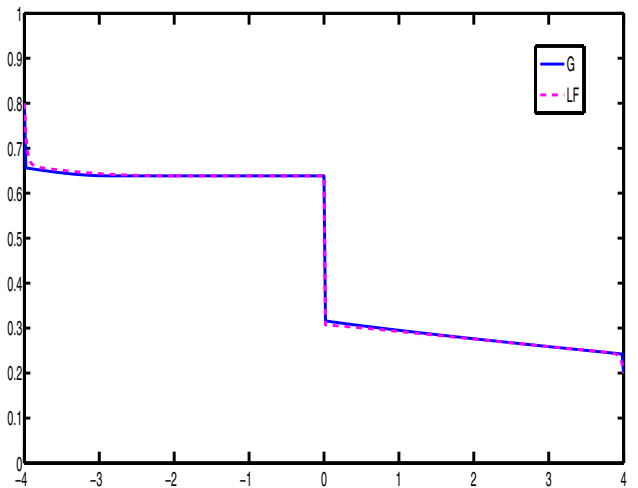

(b)

Figure 9. Solution of Experiment 2.1 at $T=1$.

Experiment 1.2. Overcompressive initial data. We take the following overcompressive data.

$$
u_{0}=\left\{\begin{array}{lll}
0.2 & \text { if } & x<0 \\
0.8 & \text { if } & x>0
\end{array}\right.
$$

We compute the solution for the above Riemann problem (5.2) with $\lambda=1 / 16, h=\frac{8}{200}$ (see Fig. 8a) and $\lambda=1 / 16, h=\frac{8}{400}$ (see Fig. 8b) at time $T=1$. Here also the figure shows that Lax-Friedrichs is more diffusive than Godunov scheme but as mesh size goes to zero both agree.

Experiment 2. Overcompressive intersection case. By interchanging $f$ and $g$ we get overcompressive intersection. In this case $g$ and $f$ becomes

$$
g(u)=\frac{20 u^{2}(1-u)^{2}}{u^{2}+2(1-u)^{2}} \quad \text { and } f(u)=\frac{50 u^{2}(1-u)^{2}}{10 u^{2}+(1-u)^{2}} .
$$

We let the connection be $\left(A, \theta_{f}\right)=(.63839972, .317014)$. With this choice of $f, g$ and connection we compute the solution for above initial datas (5.2), (5.1).

The solutions of Experiment 2 for initial data (5.1)(undercompressive data) and (5.2) (over compressive data) at time $T=1$ are presented in Figure 9 and Figure 10 respectively. Solutions in Figure 9a and Figure 10a are computed with $\lambda=1 / 16, h=\frac{8}{200}$ and whereas Figures $9 \mathrm{~b}$ and $10 \mathrm{~b}$ is computed with $\lambda=1 / 16, h=\frac{8}{400}$. Both 


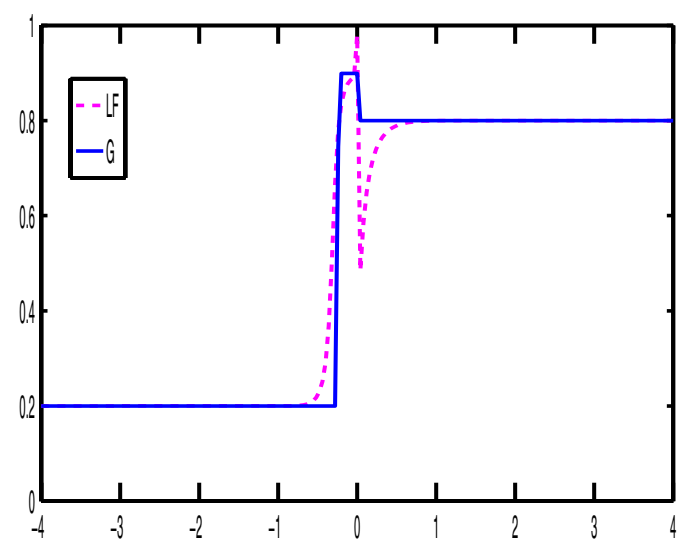

(a)

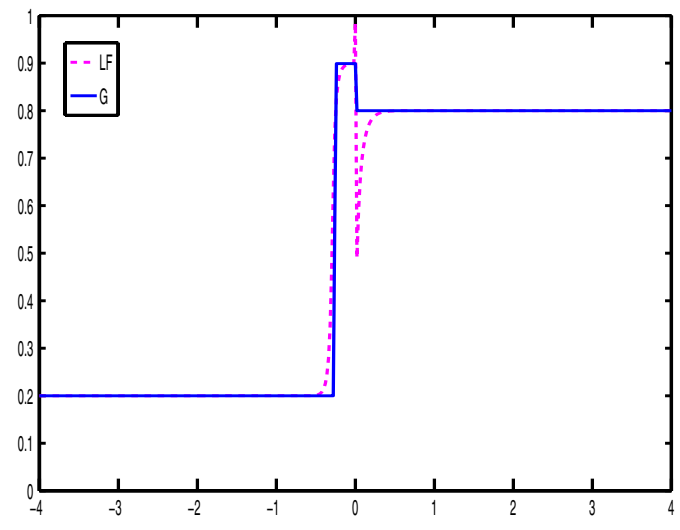

(b)

Figure 10. Solution of Experiment 2.2 at $T=1$.

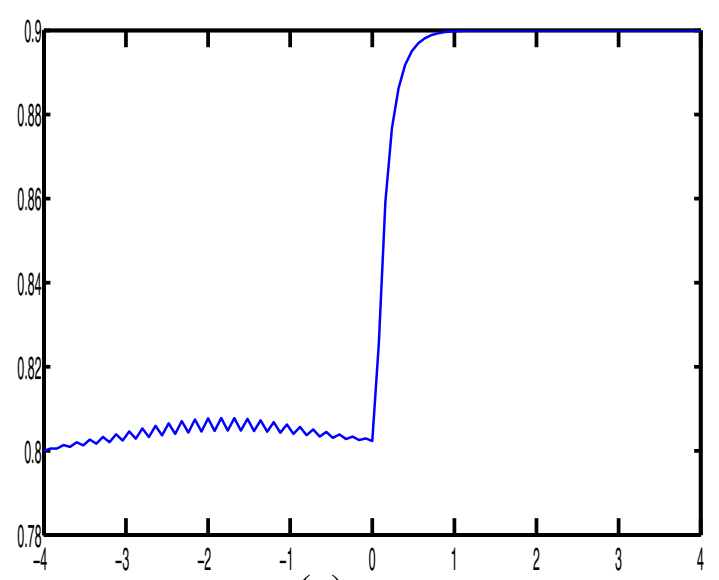

(a)

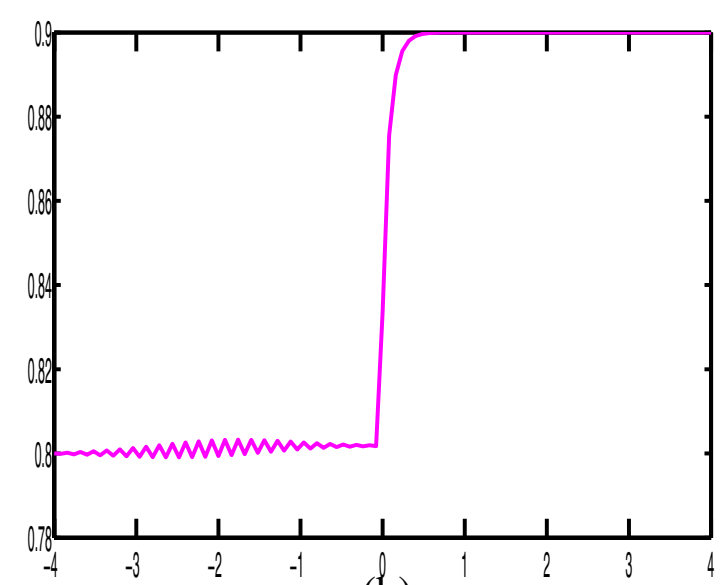

(b)

Figure 11. Left: Solution of the Lax-Friedrichs scheme given by Karlsen and Towers. Right: Solution of the Lax-Friedrichs scheme given by (3.16).

the figure shows that the Lax-Friedrichs is more diffusive than Godunov scheme but as mesh size goes to zero both the solutions coincide.

In the case of overcompressive data, the approximated solution computed by both the LF produces "spike" at the interface, see Figure 10. The appearance of the spike is due to the construction of scheme using translation. The height of the "spike" is independent of $h$ and as mesh parameter $h$ goes to zero the area, where the "spike" form, is also goes to zero.

Like in the Lax-Friedrichs scheme given in [16], when the Riemann data $(a, b)$ satisfies $g^{\prime}(a) f^{\prime}(b)>0$, here also LF produces oscillation away from the interface, for example see the Figure 11. For this we take the following initial data

$$
u_{0}(x)= \begin{cases}0.8 & \text { if } \quad x<0 \\ 0.9 & \text { if } \quad x>0\end{cases}
$$

and the fluxes are the same as in Experiment 1. In Figure 11 solution is computed for the initial data (5.3) with $\lambda=.1, h=8 / 100$ at time $T=1$. 


\section{Conclusion}

In previous works $[5,9]$ numerical fluxes to discontinuous flux with $(\mathrm{A}, \mathrm{B})$ connection for Godunov, Engquist-Osher respectively are constructed. But the construction of Lax-Friedrichs scheme for $(A, B)$ connection was left open. In this work we have generalized the construction of the interface numerical flux for $(A, B)$ entropy solution of conservation laws with discontinuous flux. From this one can construct monotone numerical schemes. In particular it includes the standard fluxes like Gudunov, Engquist-Osher, Lax-Friedrichs and Rusanov.

\section{REFERENCES}

[1] Adimurthi and G.D. Veerappa Gowda, Conservation laws with discontinuous flux. J. Math. Kyoto Univ. 43 (2003) $27-70$.

[2] Adimurthi, R. Dutta, Shyam Sundar Ghoshal and G.D. Veerappa Gowda, Existence and nonexistence of TV bounds for scalar conservation laws with discontinuous flux. Commun. Pure Appl. Math. 64 (2011) 84-115.

[3] Adimurthi, J. Jaffré and G.D. Veerappa Gowda, Godunov type methods for scalar conservation laws with flux function discontinuous in the space variable. SIAM J. Numer. Anal. 42 (2004) 179-208.

[4] Adimurthi, S. Mishra and G.D. Veerappa Gowda, Explicit Hopf-Lax type formulas for Hamilton-Jacobi equations and conservation laws with discontinuous coefficients. J. Differ. Equ. 241 (2007) 1-31.

[5] Adimurthi, S. Mishra and G.D. Veerappa Gowda, Optimal entropy solutions for conservation laws with discontinuous fluxfunctions. J. Hyperbolic Differ. Equ. 2 (2005) 783-837.

[6] B. Andreianov, K.H. Karlsen and N.H. Risebro, A theory of L1-dissipative solvers for scalar conservation laws with discontinuous flux. Arch. Ration. Mech. Anal. 201 (2011) 27-86.

[7] R. Bürger, K.H. Karlsen, N.H. Risebro and J.D. Towers, Well-posedness in $\mathrm{BV}_{t}$ and convergence of a difference scheme for continuous sedimentation in ideal clarifier thickener units. Numer. Math. 97 (2004) 25-65.

[8] R. Bürger, K.H. Karlsen, N.H. Risebro and J. D. Towers, Monotone difference approximations for the simulation of clarifierthickener units. Comput. Vis. Sci. 6 (2004) 83-91.

[9] R. Bürger, K.H. Karlsen and J. D. Towers, An Engquist-Osher-type scheme for conservation laws with discontinuous flux adapted to flux connections. SIAM J. Numer. Anal. 47 (2009) 1684-1712.

[10] Crandall, G. Michael and Majda, Andrew, Monotone difference approximations for scalar conservation laws. Math. Comput. 34 (1980) 1-21.

[11] S. Diehl, Conservation Laws with Applications to Continuous Sedimentation, Doctoral Dissertation. Lund University, Lund, Sweden (1995).

[12] S. Diehl, A conservation laws with point source and discontinuous flux function modelling continuous sedimentation. SIAM J. Appl. Math. 56 (1996) 388-419.

[13] T. Gimse and N.H. Risebro, Riemann problems with discontinuous flux function, Proc. of 3rd Internat. Conf. Hyperbolic Problems, Studentlitteratur, Uppsala (1991) 488-502.

[14] Jaffré, Jérôme and S. Mishra, On the upstream mobility scheme for two-phase flow in porous media. Comput. Geosci. 14 (2010) 105-124

[15] E. Kaasschieter, Solving the Buckley-Leverret equation with gravity in a heterogeneous porous media. Comput. Geosci. 3 (1999) 23-48.

[16] K.H. Karlsen and J.D. Towers, Convergence of the Lax-Friedrichs scheme and stability for conservation laws with a discontinuous space-time dependent flux. Chinese Ann. Math. Ser. B 25 )(2004) 287-318.

[17] C. Klingenberg and N.H. Risebro, Convex conservation laws with discontinuous coefficients, existence, uniqueness and asymptotic behavior. Commun. Partial Differ. Equ. 20 (1995) 1959-1990.

[18] B. Keyfitz, Solutions with shocks: An example of an $L^{1}$-contractive semi-group. Commun. Pure Appl. Math. 24 (1971) 125-132.

[19] S. Mishra, Analysis and Numerical approximation of conservation laws with discontinuous coefficients, Ph.D. thesis, Indian Institute of Science, Bangalore (2005).

[20] S.Mochon, An analysis for the traffic on highways with changing surface conditions. Math. Model. 9 (1987) 1-11.

[21] H. Nessyahu and E. Tadmor, Non-oscillatory central differencing for hyperbolic conservation laws. J. Comput. Phys. 87 (1990) 408-463.

[22] N. Seguin and J. Vovelle, Analysis and approximation of a scalar conservation law with a flux function with discontinuous coefficients. Math. Models Methods Appl. Sci. 13 (2003) 221-257.

[23] B. Temple and E. Isaacson,, Nonlinear resonance in systems of conservation laws. SIAM J. Appl. Math. 52 (1992) $1260-1278$.

[24] J.D. Towers, A difference scheme for conservation laws with a discontinuous flux: the nonconvex case. SIAM J. Numer. Anal. 39 (2001) 1197-1218

[25] J.D. Towers, Convergence of a difference scheme for conservation laws with a discontinuous flux. SIAM J. Numer. Anal. 38 (2000) 681-698.

[26] S. Tveit, Numerical methods for hyperbolic conservation laws with discontinuous flux. Master of Science Thesis in Reservoir Mechanics, University of Bergen (2011). 